\title{
Time-Based Air Traffic Management Using Expert Systems
}

\section{Tobias and J.L. Scoggins}

April 1986

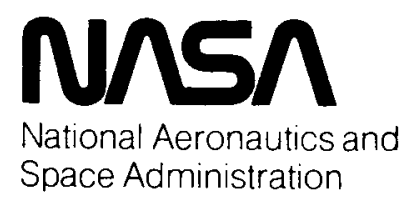


NASA Technical Memorandum 88234

\section{Time-Based Air Traffic Management Using Expert Systems}

L. Tobias,

J. L. Scoggins, Ames Research Center, Moffett Field, California -

April 1986

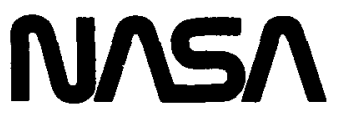

National Aeronautics and

Space Administration

Ames Research Center

Moffett Field, California 94035 


\section{SUMMARY}

A prototype expert system has been developed for the time scheduling of aircraft into the terminal area. The three functions of the air-traffic-control schedule advisor are as follows: First, for each new arrival, it develops an admissible flight plan for that aircraft. Second, as the aircraft progresses through the terminal area, it monitors deviations from the aircraft's flight plan and provides advisories to return the aircraft to its assigned schedule. Third, if major disruptions such as missed approaches occur, it develops a revised plan. The advisor is operational on a Symbolics 3600, and is programmed in MRS (a logic programming language), Lisp, and Fortran.

\section{INTRODUCTION}

At present, the decision-making process of air traffic control (ATC) in the extended terminal area (from $150 \mathrm{n}$. mi. from touchdown until touchdown) is a manual one. Controllers expertly direct traffic flow to maximize capacity without compromising safety by issuing to each aircraft clearances which specify speed, altitude, or heading requirements. In terms of arrivals to a single runway at a major terminal area, this is generally accomplished as follows: along each of the three to four major arrival directions, traffic is spaced uniformly. However, the traffic flows from the various arrival directions are uncoordinated. It is the responsibility of the final controller to take these uncoordinated flows and establish a sequence of traffic which does not violate separation constraints between consecutive aircraft.

The automation of selected ATC decision-making functions using a time-based framework has been the subject of a series of real-time simulation studies (1). The goals of these studies have been to increase landing rate and to reduce fuel consumption, and in addition, to reduce controller workload. Time scheduling is to be accomplished by developing a time plan, and a means of controlling aircraft to achieve a desired time, so that traffic flows which reach the final controller are coordinated, and that little maneuvering is required to achieve the final sequencing.

The key element in the time-based system is a four-dimensional (4D) algorithm (2) which, given the present position, route, and touchdown time, generates a timetable of commands to control the aircraft to follow the route specified and touchdown at the specified time. Flight tests of aircraft equipped with the 4D software have demonstrated that accuracies of $\pm 5 \mathrm{sec}$ can be obtained. In addition, it has 
been demonstrated in simulation studies (3) that modifications of the 4D algorithms can be used by the ATC computer to generate a speed advisory to assist in achieving time accuracies of $\pm 20 \mathrm{sec}$ for aircraft which are unequipped. 1

Real-time simulation studies have used these 4D algorithms to investigate the feasibility of a time-based set of candidate operational procedures for handling a mix of 4D-equipped and -unequipped aircraft in the terminal area. The operational procedures include a time-assignment procedure for new arrivals, a procedure to correct for time errors as the aircraft progresses through the terminal area, and a means of handling missed approaches. Results indicate that the procedures investigated are promising; however, they represent only a limited (but important) subset of the procedures which will be required for implementing a time-based system. Attention needs to be focused on a more complete, adaptable plan for scheduling traffic into the terminal area.

This paper describes the initial step in implementing a more complete, timebased system for traffic management in the extended terminal area using the framework of expert systems. A prototype schedule advisor formulated as an expert system is described. First, the rationale for an expert systems approach is given. This is followed by a detailed description of the ATC problem. The software design is then outlined, with a complete, documented code given in the appendix. Finally, plans for the continued development of the schedule advisor are discussed.

\section{RATIONALE FOR AN EXPERT SYSTEMS APPROACH FOR A SCHEDULE ADVISOR}

The plan for implementing a schedule advisor in an actual operating environment will be initially to provide a system with limited capabilities, and to gradually increase the sophistication of the computer software as more of the controller's expertise is understood. Thus, the controller would initially possess considerable knowledge not resident in the advisor, and would need to interact with the advisor to modify or override the computer-generated plans. The controller may want to prohibit the scheduling of aircraft during a time interval because of runway visibility problems, to assign priority to one aircraft because it is low on fuel, or to request a particular landing order for a group of aircraft to increase the landing rate.

In addition to the interaction requirement, it is also necessary for the advisor to explain its actions to the controller. ${ }^{2}$ The controllers may initially be skeptical of the computer-generated plans, particularly if the computer solution is

\footnotetext{
${ }^{1}$ Without speed advisories, time accuracies of $\pm 2 \mathrm{~min}$ have been achieved by contrgllers in the en route area.

The explanation capability of expert systems in the ATC context is discussed in Ref. 4.
} 
not in complete agreement with their own intuitive plan. If the logic of the computer plan is understood, the controller will gain confidence in the system more quickly.

It is also expected that the initial set of heuristics will need to be modified. The new heuristics can readily be formulated as added or modified rules: for example, the controller may wish to add a rule to restrict the speed of an aircraft which is in a given altitude range of a given sector under specified traffic flow conditions. It is important that the modifications described be readily implemented.

It is for these primary reasons--the need for a software system which is interactive, which can explain its behavior, and which can easily accept procedural modifications--that an expert systems approach has been formulated. In addition, it should also be noted that there are many other suggested expert system guidelines (5) that are met for this problem area. There are many domain experts that understand and operate the present ATC system, and there are some whose expertise has been built over a long period of time who can commit a substantial amount of time to the development of the system. In addition, the problem domain is well bounded--the problem under consideration is restricted to routes in a major terminal area from $120 \mathrm{n}$. mi. from touchdown until touchdown. At any one time the number of aircraft in the plan is less than 100, and thus reasoning about aircraft schedules in real time should not be a problem.

\section{THE PROTOTYPE SCHEDULE ADVISOR: THE ATC PROBLEM}

A prototype ATC Schedule Advisor has been developed, and will now be described. First, the ATC problem will be presented, followed by a discussion of the system design.

\section{A plan for new arrivals}

The terminal area under consideration, shown in Fig. 1, is based on the route structure in a $150 \mathrm{n}$. mi. radius of Denver, Colorado. The Denver route structure is a "four-corner-post" system with conventional jet aircraft arriving from either Drako from the northwest, Keann from the northeast, Kiowa from the southeast, and Byson from the southwest. A route for low-speed, general-aviation traffic which emanates from Colorado Springs is also considered. It is assumed that Instrument Flight Rules (IFR) prevall, and that all landings are at the Denver Stapleton Airport on runway 26L. The area under consideration contains the Denver Terminal Radar Approach Control (TRACON) and a portion of the Denver Air Route Traffic Control Center (ARTCC).

Conventional jet traffic enters the terminal area region at one of the feeder fixes of the four corner posts described, and general-aviation traffic enters from Colorado Springs. When the traffic enters the terminal area, a time assignment is generated for the aircraft to reach touchdown. The time assignment is based on the 
range of feasible times for the aircraft to fly the route. The aircraft has a preferred nominal time, but can speed up or slow down within a specified range.

There is an additional FAA requirement in which consecutive aircraft must be separated by a minimum distance; this distance is a function of the weight categories of the aircraft. Three categories have been defined: light, large, and heavy. For the route system under consideration, light aircraft are restricted to the Colorado Springs route, while both large and heavy aircraft can fly on the four jet routes. These distance-separation requirements can be translated into minimum separation times for the purpose of time scheduling (1). Thus, scheduling new arrivals involves both determining the range of feasible times for the aircraft and checking that the proposed schedule does not conflict with previously scheduled aircraft. In the prototype design, the schedule of aircraft which have arrived earlier are considered fixed, and the plan for a new arrival is based on meeting separation constraints with respect to these schedules. It may be possible that no conflict-free time exists. In this case, the new arrival is scheduled for later departure and will hold at the route entry point (feeder fix) until its assigned time.

A typical situation is shown in $\mathrm{Fig} .2$, which is a time-line representation of all the aircraft currently in the system at time 12:37:03. A separate line is drawn for each of the arrival routes previously discussed, and there is also a line for the missed-approach route, which will be discussed. On the Keann route, two aircraft are shown which have been scheduled; PA003, for which time at touchdown is 12:46:10, and UA003, which directly follows it at 12:49:05. Suppose that at time 12:37:03, WA374 reaches the feeder fix at Drako. The following times are computed by the planner: nominal time to touchdown is $12: 52: 38$, with a range of $12: 51: 38$ to $12: 54: 38$. Any time assignment outside this range would necessitate some holding at Drako. The planner first attempts to schedule WA374 at the nominal, then tries other times on either side of the nominal until the range limits are reached. In this example, the aircraft can be assigned at its nominal time, and no initial holding is necessary. Holding is permitted at some interior points in the region, but interior holding is not part of the initial schedule plan.

\section{Correcting minor schedule deviations}

After creating an initial schedule plan, the next function of the schedule advisor is to monitor each aircraft as it proceeds from feeder fix to touchdown, detect deviations from its assigned $4 \mathrm{D}$ route, and suggest corrective actions. It is important to note that, while advisories can perhaps be generated continuously in real-time operation, the number of corrective actions (i.e., clearances based on computer-generated advisories) must be kept small. There is still a substantial research problem to optimize the number of advisories, the type of advisories, and the times in which they should be given to the aircraft to minimize the deviation from scheduled touchdown time. One time of advisory, the speed advisory, has been mentioned earlier. In the prototype schedule advisor, the only advisories used are heading and path advisories, which will now be discussed. 
When the aircraft departs the feeder fix, a planned touchdown time has been established. A 4D route is synthesized and used as the reference trajectory as the aircraft proceeds from feeder fix to touchdown. To correct for minor deviations from the 4D route, a decision point has been established on each of the routes. Figure 3 shows the decision point for the two northern routes. When the aircraft reaches the decision point $\mathrm{D}_{\mathrm{K}}$ on Keann, or $\mathrm{D}_{\mathrm{D}}$ on Drako, a path correction is generated so that the aircraft can meet its assigned time schedule. From Drako, the aircraft will be required to adjust its time to turn, while from Keann, a heading change will be required. Note that the advisory will include a warning if, even with the largest correction allowed, an error remains. In the prototype system, no attempt is made to resolve this type of problem. At present, it is necessary for the controller to call for a missed approach, or take some other action to resolve the problem.

\section{Schedule revisions}

So far, only minor deviations have been considered, and the strategy has been to generate corrections to return the aircraft to its assigned time so that the schedule plan for other aircraft need not be altered. However, the schedule advisor must consider the situation when major disruptions occur, i.e., when it is not possible to retain the existing plan. These changes may be necessitated by aircraft executing a missed approach or an emergency landing, or by weather-related modifications such as rerouting to avoid severe weather, runway closure, or runway use changes caused by shifting winds. A revised plan must be generated which must handle the disruption in a continually safe manner, and which will return to an expeditious flow after an initial transient period. In the prototype system, two classes of major disruptions are considered: missed approaches and runway closures.

If an aircraft is to execute a missed approach (Fig. 4), it proceeds along its route in level flight toward the touchdown point, then to the departure end of the runway, and then turns right heading toward point $\mathrm{H}_{\mathrm{D}}$ where it rejoins the Drako route for a second attempt at a flight to touchdown. A new touchdown time must be assigned for this aircraft, and it is preferred that the missed-approach aircraft be given priority to land at its earliest possible time. This may necessitate holding other aircraft at the feeder fixes or the internal holding points until a revised conflict-free touchdown time is available.

A sample holding situation is illustrated in Fig. 5. Figure 5a shows a timeline plot at time 13:06:54. At this time, the controller decides that AA251 needs to execute a missed approach. The missed-approach command is entered into schedule advisor, and the revised time line shown in Fig. $5 b$ is generated. The aircraft AA251 now appears on the missed-approach line, and has been rescheduled without holding.

Elements of a plan for handling runway closure have been developed. Suppose that the controller declares that a runway is closed for the interval $\left(t_{i}, t_{f}\right)$, where $t_{i}$ is the time when the closure is first effective, and $t_{f}$ is the time when the runway is to be reopened. 3 The advisor first determines which flights are currently assigned in the blocked interval. These flights and any flights which 
follow them are sequentially rescheduled. Any flight which has passed the last holding point will be told to execute a missed approach and proceed in for a new approach or for holding as required.

A sample runway closure situation is shown in Fig. 6. Figure 6a shows a time line plot at 13:20:16. At this time the controller decides that no aircraft can land in the interval from now until 13:23. One aircraft, PA35, is scheduled to land in this interval. The revised plan is shown in Fig. 6b. No aircraft are scheduled in the blocked interval. The aircraft PA35 has executed a missed approach and is now rescheduled without holding.

\section{THE PROTOTYPE SCHEDULE ADVISOR: SOFTWARE DESIGN}

A prototype for the ATC schedule advisor has been programmed on a Symbolics 3600 Lisp machine. A fully commented program listing is given in the appendix; this discussion serves to highlight the key features of the software involved. The intention was to combine existing numerical subroutines, capable of $4 D$ planning single flights, with artificial intelligence (AI) planning techniques to produce an intelligent 4D scheduler for a system of flights. In addition, this scheduler will serve as a research tool, with the ability to add new features as they are developed.

\section{Problem representation}

A generalized representation of the problem is to treat the terminal area as a directed graph. A sample graph is given in Fig. 7. Each node on the graph represents some physical point such as touchdown or a feeder fix. These nodes are labeled " $a$ " through " $k$ " in the figure. Each directed arc represents a transition in terms of the time required for a flight to move between the two connected nodes. For example, between the feeder $f i x$ " $j$ " and the waypoint " $f, "$ a flight may have the option to wait at the holding point labeled " $h . "$

Various information is maintained for each arc, such as the distance to the runway, alternate paths, and procedures that must be executed whenever a flight enters or exits the arc. For example, if a given arc represents the travel between a decision point and the runway, the arc's entry procedure would then include a code to generate a path correction.

Two ordered lists are also maintained for each arc which denote, respectively, the flights that are scheduled to enter and exit the arc. In Fig. 7 , the flight PA35 is scheduled to enter at 13:02:23 and then exit at 13:11:45. Upon exiting, PA35 should be flying at an altitude of $36,000 \mathrm{ft}$ and a calibrated airspeed of

${ }^{3}$ The case when the runway is closed indefinitely is more complex and is not considered in the prototype system. 
182 knots. Likewise, the flight AA24 has already entered the arc at $12: 50: 33$ and is scheduled to exit at $12: 59: 57$.

This information shows the planned state of the arc at any given time, and can be used to help reschedule flights and to detect potential conflicts. Also, the system can use the list of entering flights at the touchdown arc to determine possible "time slots". where aircraft can be allowed to land. Given this representation, the problem is to develop a planner which finds good time-based paths through the graph for flights that need to be scheduled, and to develop a control structure which operates on the graph as events occur (i.e., flights enter and exit arcs, plans are revised, etc.).

\section{Planning functions}

The first planning goal is to always have a consistent plan for the overall system. Each flight in the system must always have a scheduled path from its current position to touchdown. This path consists of a list of scheduled waypoint arrival times which cannot conflict with blocked-runway time intervals or separation requirements for other flights. If situations arise in which the system cannot adequately plan for all of the arriving flights, some of the air traffic will then be placed in holding patterns. For example, if the runway is blocked and a number of flights arrive at the feeder fixes, then some of the flights may have to hold until conflict-free touchdown times can be scheduled.

Next, consider what kind of information the planner must understand and maintain for each flight. This is determined by the numerical routines for 4D path generation and control which lie at the heart of the planner. Input parameters to these routines consist of current time, altitude, calibrated airspeed, distance to touchdown, and speed profile. Output parameters from these routines consist of a list of the computed altitude, airspeed, and distance for the flight at various time steps to touchdown, and the controller advisories required to execute the plan.

The planner must determine a good strategy for a given flight, feed the correct parameters to the numerical routines, and check the resulting plan for any conflicts with flights that are already scheduled in the system. The strategy determines whether a flight should hold at the feeder fix, or use a faster or slower speed profile, etc., and hence determines the parameters to the numerical routines. Plan segments that could be added to arcs on the graph are interpolated from the time steps of the computed flight parameters. Then the planner checks for separation conflicts, blocked-runway conflicts, etc., to test the candidate plan along its path through the graph. This plan is returned if valid; otherwise, the planner backtracks to attempt some alternate strategy.

The planner therefore uses a "generate and test" structure, backtracking whenever some conflict is found. Logic programming is used to encode the planner since backtracking is an inherent feature. Flight strategies can also be represented symbolically and reasoned about conveniently. Heuristics can be added to guide the backtracking. As more engineering knowledge is applied to the ATC problem, more 
expertise will be encoded into these heuristics to make the path-generation process more efficient.

\section{System architecture}

The schedule advisor runs in the Symbolics Zetalisp environment using software programmed in MRS, Lisp, and Fortran. The Lisp functions call a Fortran program to run when the numerical subroutines are needed, and parameters are communicated through input and output data files. The numerical subroutines for $4 \mathrm{D}$ path generation and control consist of nearly 3000 lines of a Fortran 77 code originally developed under VAX VMS (3). This code performs a Runge-Kutta integration to determine optimal descent paths. The Symbolics software provides a Fortran 77 development environment and only a few minor changes were needed to port these routines. For instance, the Symbolics Fortran had a shorter line length, and exclamation points could not be used for in-line comments.

Combining Lisp and Fortran code into the same software system produces some challenges. Fortran requires a main program to run before any subroutines can be called, which restricts the use of Fortran subroutines within Lisp programs. To avoid this, the software can be run as a Fortran main program with Lisp functions called as subroutines. Fortunately, data types such as characters, integers, and reals tend to be machine-independent; hence, parameter passing does not require any coercion. Another option chosen for this system is to run Fortran routines as separate programs, or even separate processes, and have Lisp and Fortran communicate through data files or input/output buffers.

One interesting point is that Symbolics Fortran uses Lisp as the intermediate code during compilation, and that it retains the user-defined Fortran variable names for the corresponding Lisp symbols in the intermediate code. This is unfortunate since Fortran programmers tend to use the real variable " $T$ " to denote time values, but the name "T" in Lisp denotes a system-wide symbol for truth. For this system, it was sufficient to rename all Eortran "T" variables as "T1."

Most of the general programming has been Jone in the Symbolics Lisp language called Zetalisp, which is very close to Common Lisp. This has proven to be a very powerful and well-designed programming language. One major feature is that Zetalisp has a good set of software tools available for writing and debugging code, such as the ZMACS screen editor.

Zetalisp also provides several useful packages, such as TV for windows and graphics, and Flavors for object-oriented programing. The user interface, programmed using the TV package, consists of three windows and various menus as shown in Fig. 8. A "Time Routes" window shows a graphic depiction of the time-scheduled flights in the terminal area. An "Advisories" window displays advisories and expectations about the descending flights. A "Listener" window gives the user access into the system by providing a command prompt and a mouse with sensitive pop-up menus. 
Objects, defined with the Flavors package, are useful for representing data structures and for representing knowledge about the system which changes frequently or involves "inheritance." For example, an object is created for each flight that enters the system. Various slots are defined for each flight object, such as "touchdown time," or "neighboring flights." Inheritance is useful in defining classes of objects. For instance, a general class of objects called PLANE could have attributes such as "scheduled touchdown time" "neighboring flights," which all aircraft must have values for. Another type of objects could be defined for HEAVYAIRCRAFT. As a subclass of PLANE, these would inherit all the properties of PLANE objects, and possibly add other more specific attributes, such as "separation requirements for heavy aircraft."

Other forms of knowledge about the system are represented in MRS, which provides logic programming similar to the Prolog language. MRS, which stands for Metalevel Reasoning System, was developed by Genesereth (6)..$^{4}$ Logic programming is generally more straightforward than algorithmic methods, so long as the problem can be formulated efficiently in predicate logic. Essentially, the steps are to 1) encode the specifications for a given problem solution, 2) assert the relevant facts into the knowledge base, and 3) query a theorem prover to determine some specific solution. One advantage of formulating ATC planning in logic programming is that the rules can be easily modified and amended as the expertise involved in air traffic control becomes better understood. Another advantage is that the resulting facts and rules can be viewed as the specifications for an optimized version of the system.

MRS has features for solving problems with backward chaining, forward chaining, condition-action rules, and more. In addition, MRS automatically backtracks to search for solutions, and meta-level rules can be defined to order this backtracking (i.e., to incorporate heuristic searches). The planning functions required in this system rely on heuristic techniques since a real-time advisor cannot afford to spend very much time generating alternative $4 \mathrm{D}$ paths.

Facts which do not change over time, such as "Flight XX007 executed a missed approach at time 11:30:45," or "Waypoint WP3 is a feeder fix for the KIOWA route," are stored in the MRS knowledge base, along with the various rules. To handle a new arriving flight, the rules determine a good, feasible flight plan in the following order:

1. Use heuristics to develop a possible strategy

2. Call numeric subroutines to compute a plan from this strategy

3. Check constraint rules to test the plan for violations, blocks

${ }^{4} \mathrm{An}$ earlier version of the schedule advisor by B. Boesch, D. Gregory, J. L. Scoggins, and L. Tobias was implemented entirely in MRS as a class project in Building Expert Systems at Stanford University. 
Failure to satisfy the constraint rules at any point will invoke backtracking to search for an alternative plan.

\section{Program operation}

Assume that the schedule advisor software runs on a personal workstation networked to host computers for real-time data access. Actual operation of the system basically differs from simulation by the source of the data. Using the Symbolics interface facilities, which provide multiple graphics windows and user interaction menus, the user can create new arrivals, block runways, alter the data structures and knowledge base, execute scenario data files, and step or reset the system clock.

The main program executes a loop to

1. Look for and process input data

2. Update the system clock

3. Check the graph for events that should have occurred by the new current clock time and execute them

At any time, the user can activate a mouse button to interrupt the main loop and request a command menu. Most of the commands are used for data input, such as creating new arrivals, signaling a missed approach, or blocking the runway for some time period. Some other commands provide system controls, such as regulating the Lisp machine's memory garbage collection, or resetting the system clock.

To create a new arrival, for instance, the user selects the "New Arrival" menu option. A special menu then prompts for the flight identification number, type of aircraft, arrival feeder fix, estimated arrival time at the feeder fix, etc. A new object is created to represent this flight, and the planning begins. The resulting advisories are displayed, and the aircraft symbol is plotted on the time line.

\section{CONCLUDING R_MARKS}

A prototype schedule advisor formulated as an expert system has been developed to assist the air traffic controller in the time scheduling of traffic in the extended terminal area. The advisor develops a time plan for all new arrivals into the terminal area, which takes into account the aircraft performance requirements and the schedules of other aircraft which have already entered into the terminal area. The advisor monitors aircraft flight progress through the terminal area and generates advisories (candidate ATC clearances) to the controller to correct deviations from the time plan. Some initial procedures for revising the plan to accommodate missed approaches and blocked runways have been included.

Future research will be focused on refining the advisor, i.e., applying knowledge of engineering techniques to develop better planning heuristics. The rules 
developed so far basically demonstrate what types of reasoning can be done, without attempting to be detailed, or to be very precise. This knowledge engineering would depend on a better understanding of the problem domain through:

1. ATC expertise

2. NASA research through simulation, field testing, etc.

3. Encoded domain policies and conventions, e.g., FAA regulations

Among the general issues that need to be addressed in refining the schedule advisor are flight-path optimization, procedures to minimize time deviations at key schedule points, and strategies to improve airport capacity. For example, when planning new arrivals, one question to address would be whether to hold a flight for some time and have it follow a fast speed profile, or to have it just follow a slow speed profile without holding. Another question would be to switch the landing order of scheduled flights as new flights arrive.

As far as the system architecture is concerned, the next step would be to distribute the advisor. For example, it would be good to have both the numerical subroutines and the graphic display windows run as spearate processes. This could alleviate some of the problems found in combining various programming languages in the same run-time environment.

The overall system should be represented in terms of standard data structures, such as directed graph, priority queues, etc. The logic programming would then be used exclusively for the planner; that is, to generate and test strategies and represent search heuristics. 


\section{References}

(1) Tobias, L., Erzberger, H., Lee, H.Q. and O'Brien, P.J.: "Mixing FourDimensional Equipped and Unequipped Aircraft in the Terminal Area," AIAA Journal of Guidance, Control and Dynamics, Vol. 8, No. 3, p. 296: May-June 1985.

(2) Lee, H.Q. and Erzberger, H.: "Time-Controlled Descent Guidance Algorithm for Simulation of Advanced ATC Systems," NASA TM-84373, 1983.

(3) Erzberger, H. and Chapel, J.D.: "Ground Based Concept for Time Control of Aircraft Entering the Terminal Area," Proceedings AIAA Guidance and Control Conference, Snowmass, CO, Aug. 1985.

(4) Cross, S.E.: "Model-Based Reasoning in Expert Systems: An Application to Enroute Air Traffic Control," Proceedings AIAA/IEEE Sixth Digital Avionics Systems Conference, Baltimore, MD, Dec. 1984.

(5) Prerau, D.S.: "Selection of an Appropriate Domain," AI Magazine, Vol. 7, No. 2, p. 18: Summer 1985.

(6) Genesereth, M.R. and Ginsberg, M.L., "Logic Programming," Communications of the ACM, Vol. 28, No. 9, p. 933: Sept. 1985. 
APPENDIX

PROGRAM LISTING 


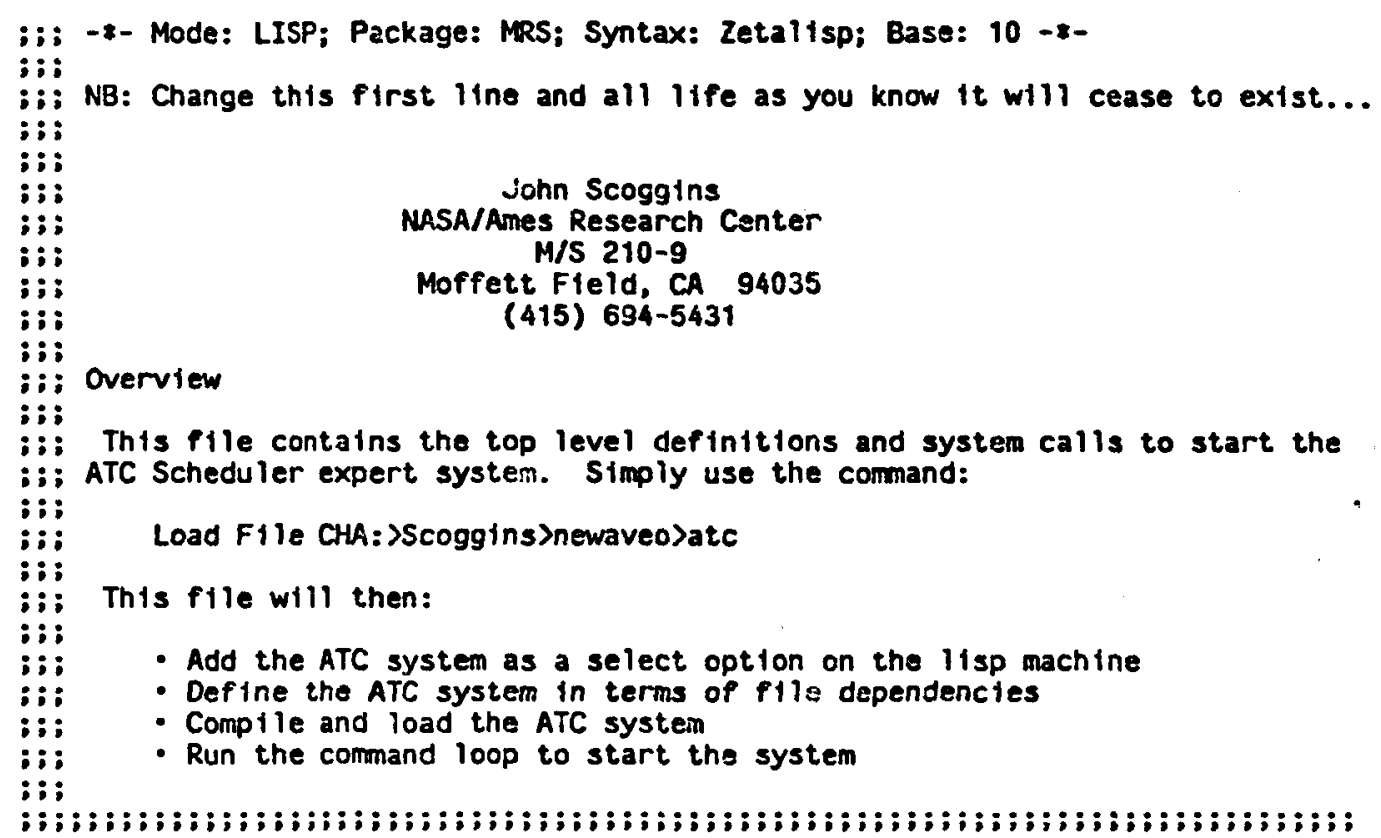

; Define the system in terms of the required flles and which ; compliers they use

(defsystem ATC-Scheduler

(:name "NASA A1r Trafflc Control Scheduler") ;System names and defaults (: short-name "ATC Scheduler")

(:pathname-default "CHA: SScoggins >newaveo)") (:package MRS)

(:module fonts ("atcfont"))

(:module pathgen ("pathgen"))

;Module names and files

(:module objects ("user" "graph"))

(:module planner ("planner"))

(:load-bfd fonts) :Compller dependenctes

(: fortran-conplle-load pathgen)

(: complle-load objects)

(:readflie planner)

; Complle and load the system

(make-system 'ATC-Scheduler

:complle

:noconfirm

)

; Call the command loop for FRAME to run the system

(send FRAME :command-1oop)

(Comment (process-run-function

"ATC Scheduler"

- (lambda ()

(send FRAME :command-loop)

)

)

) 


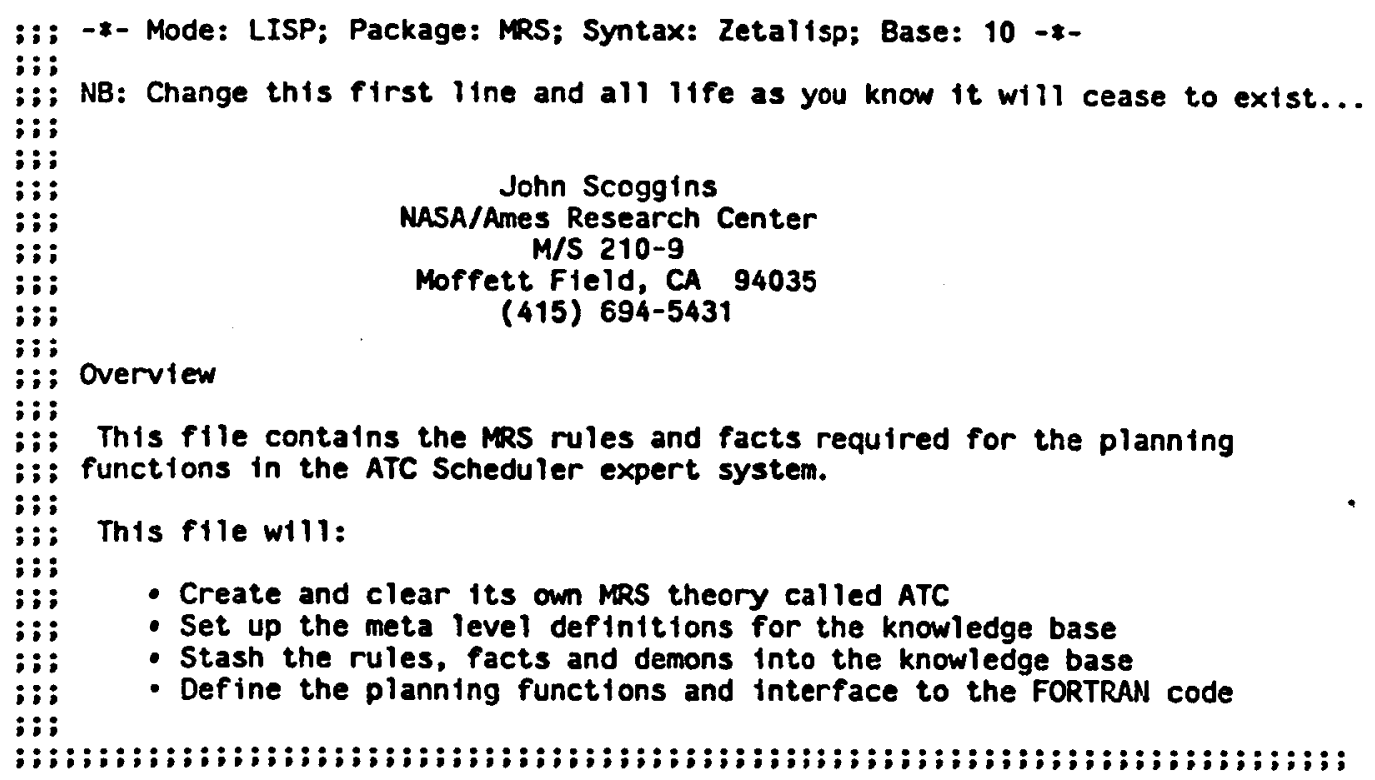




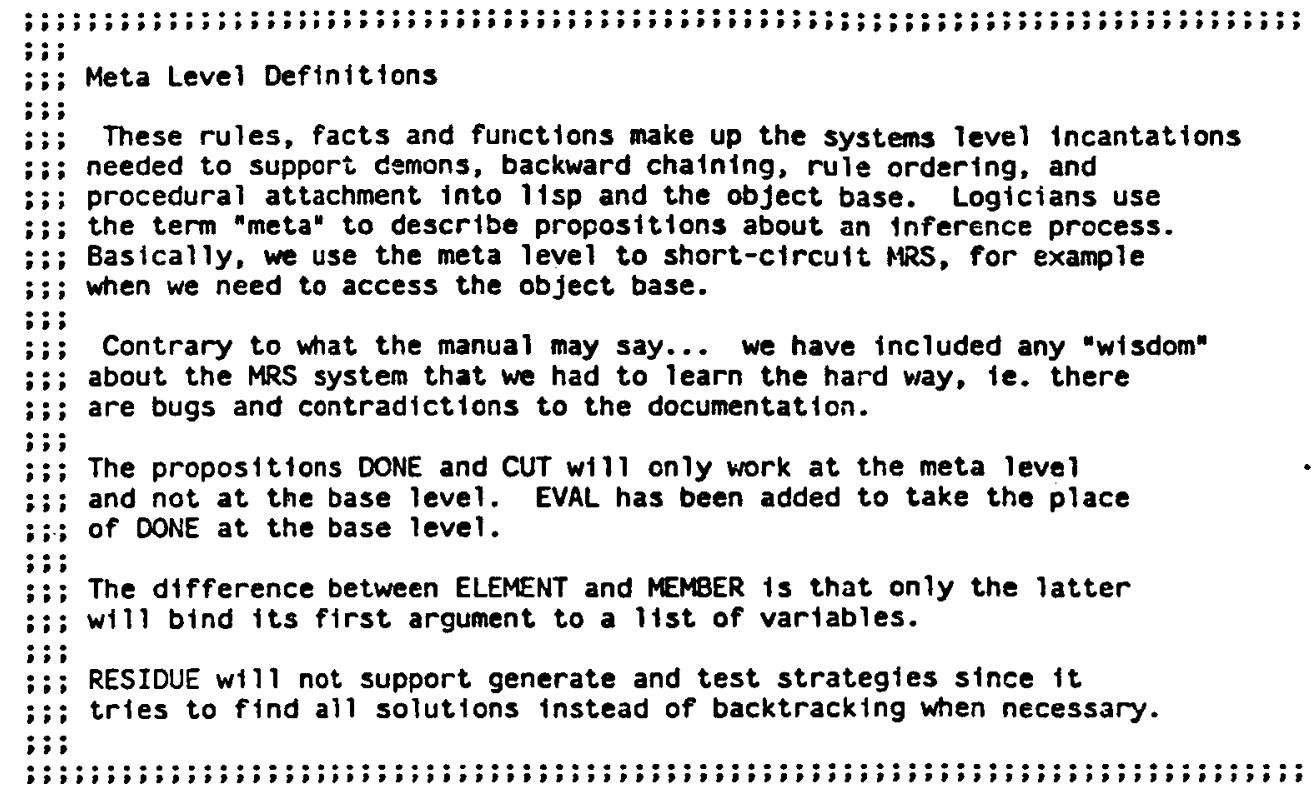

(seta theory 'GLOBAL)

(unstash '(toassert (and . 21) assert-and))

(stash "(toassert \&p $f c)$ )

(stash '(toassert (and . \$1) assert-and))

(seta theory 'ATC)

(empty theory)

; (load "CHA: >mrs)set")

(stash "(tolookup (EVAL \&funct . \&args) fq-10okup))

(stash '(tolookups (EVAL \&funct . Eargs) fq-lookups))

(stash '(11sp EVAL EVAL)) irepalr meta level to forward chain ;with more than one conclusion - this ; enables explanations for the demons

; define a theory and clear it each tima iwe reload the file - also load any inecessary MRS packages, 1tke SET

iprocedural attachment to call ; LISP and FORTRAN routines from iMRS

; Short cuts to call the most commonly need LISP functions without using EVAL - see ; the "Complete Gurde to MRS" under procedural attachment

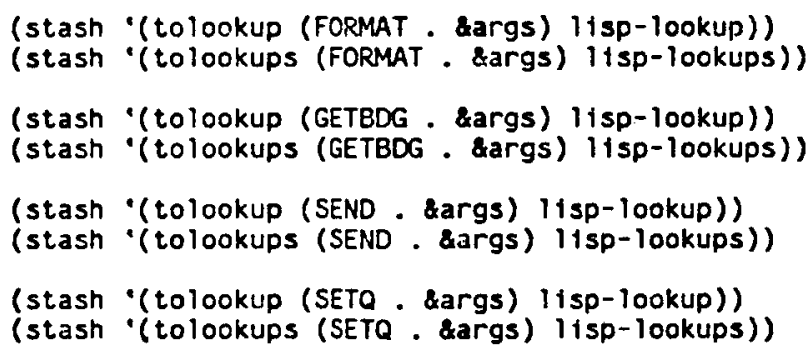

(p)

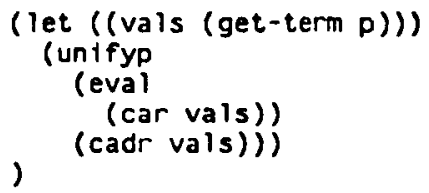

;MRS has FQ-LOOKUPS defined to do this

; kind of procedural attachment, but it ; really only works for the numerical ifunctions. LISP-LOOKUP(S) replaces the ; the EVAL proposition defined in RULES. LISP iThis is called a "computable representation" isee the flle CHA: MMRS sefrefn.lisp

;MRS requires a pluralized form for each ; computable representation 


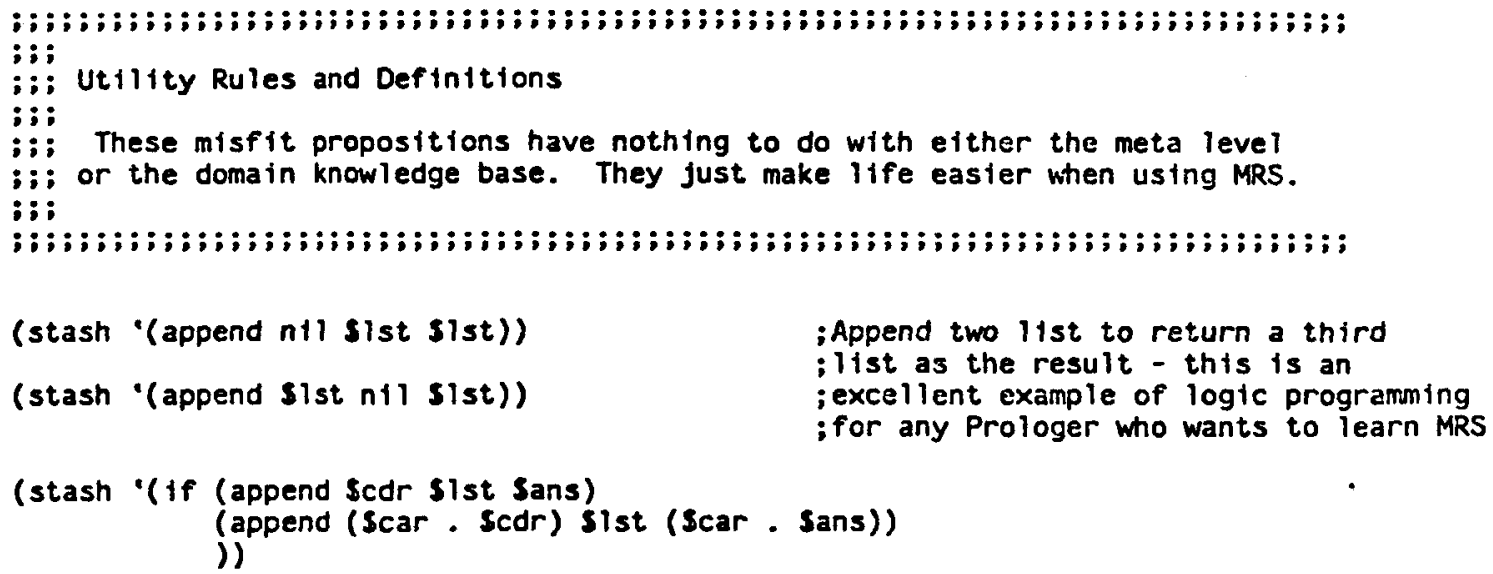




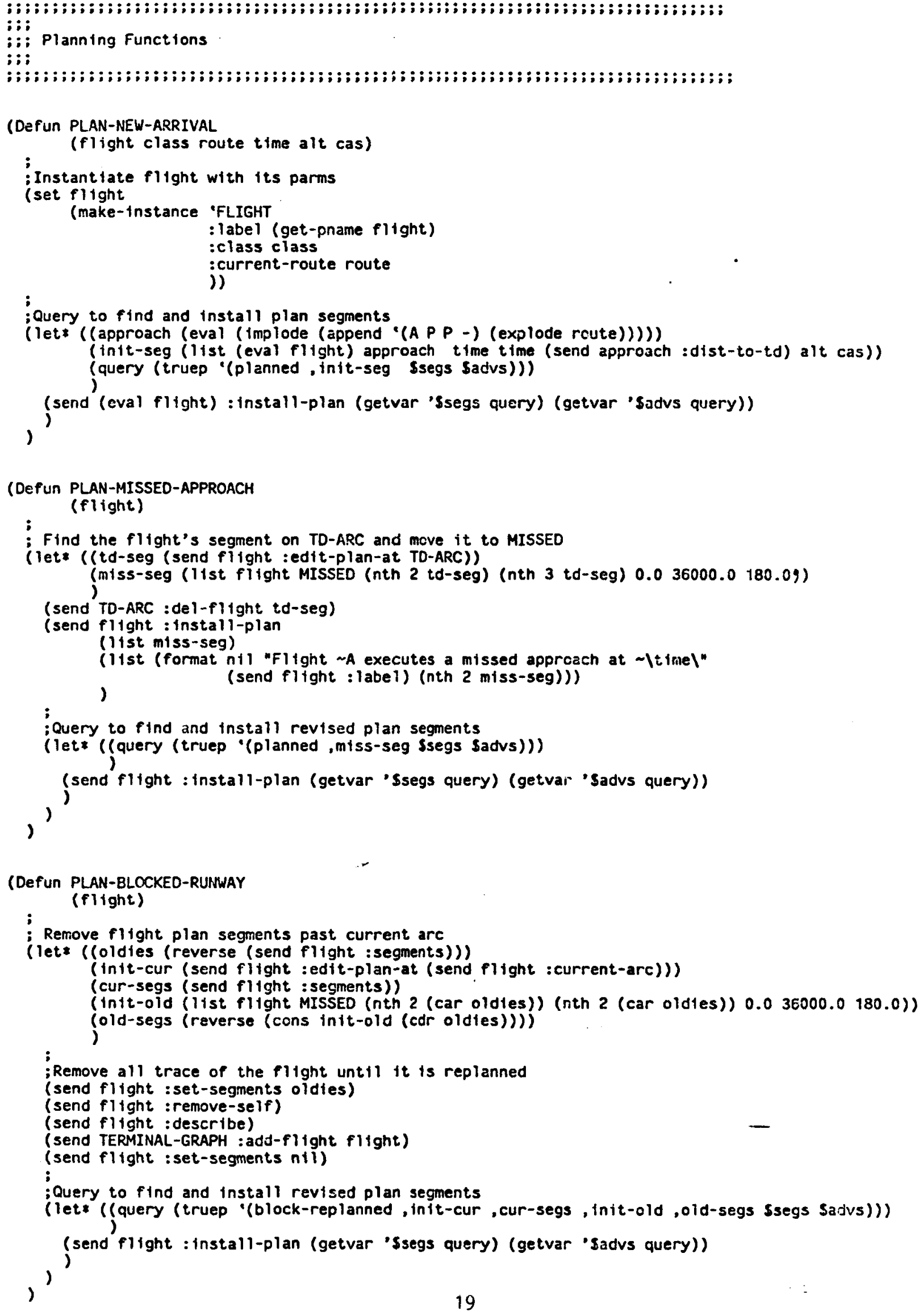




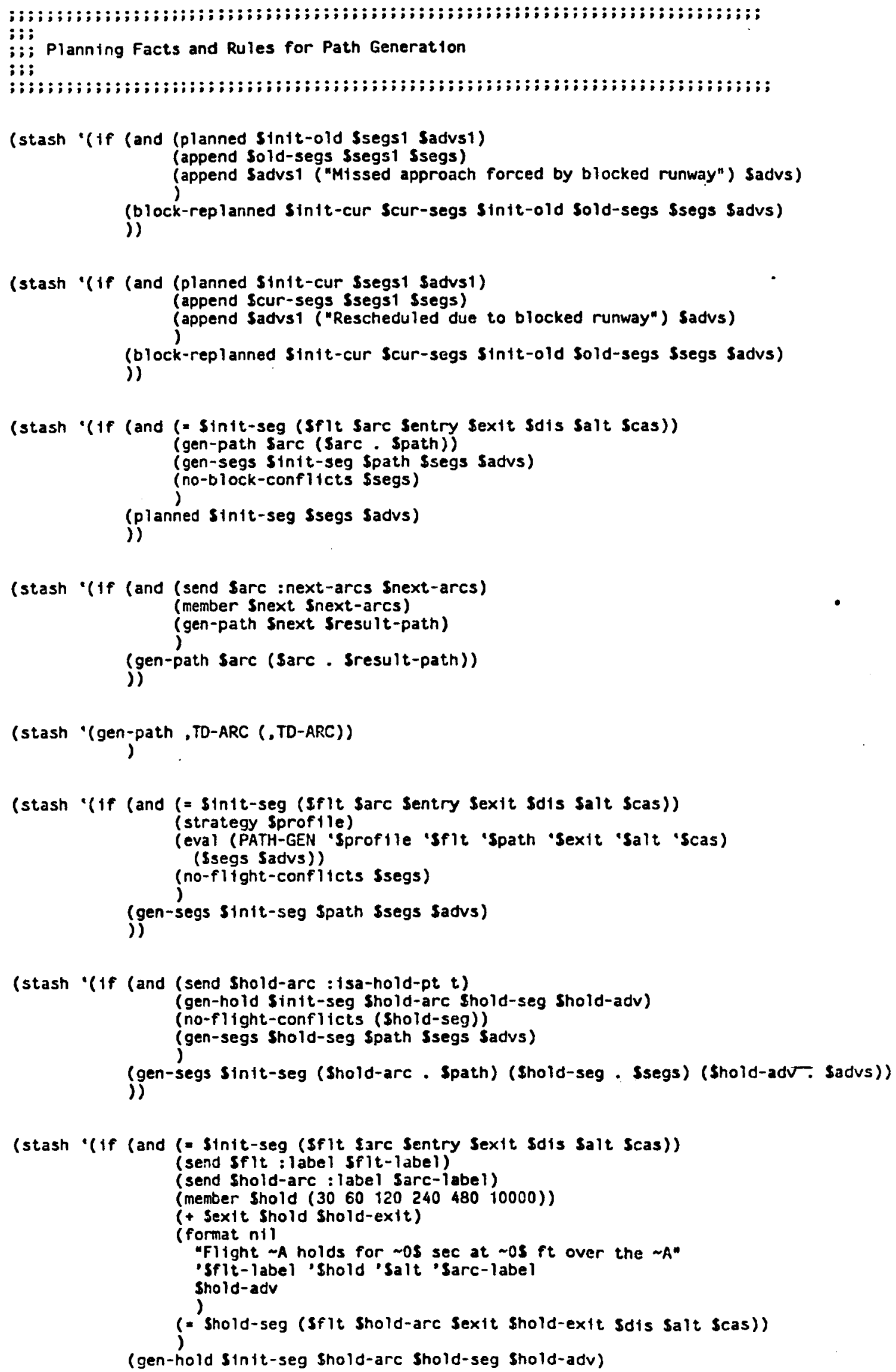


)

(stash ' (if Lunprovable

(and (member Sthis-seg \$segs)

(= (Sfit Sarc Sentry Sexit Sdis Salt Scas) Sthis-seg)

(send Sarc :wtll-conflict' Sthis-seg (Sfront sback))

(+ Sfront sback Sconflict)

() Sconflict 0 )

))

(no-f)ight-conflicts Ssegs)

))

(stash ' (if (unprovable

(and (member (Sflt, TD-ARC Sentry Sexit Sdis Salt Scas) Ssegs)

(blocked-runway St1 St2)

(< St1 Sentry St2)

i)

(no-block-conflicts Ssegs)

))

(stash "(strategy FAST)

;unt1) FORTRAN is debugged... 


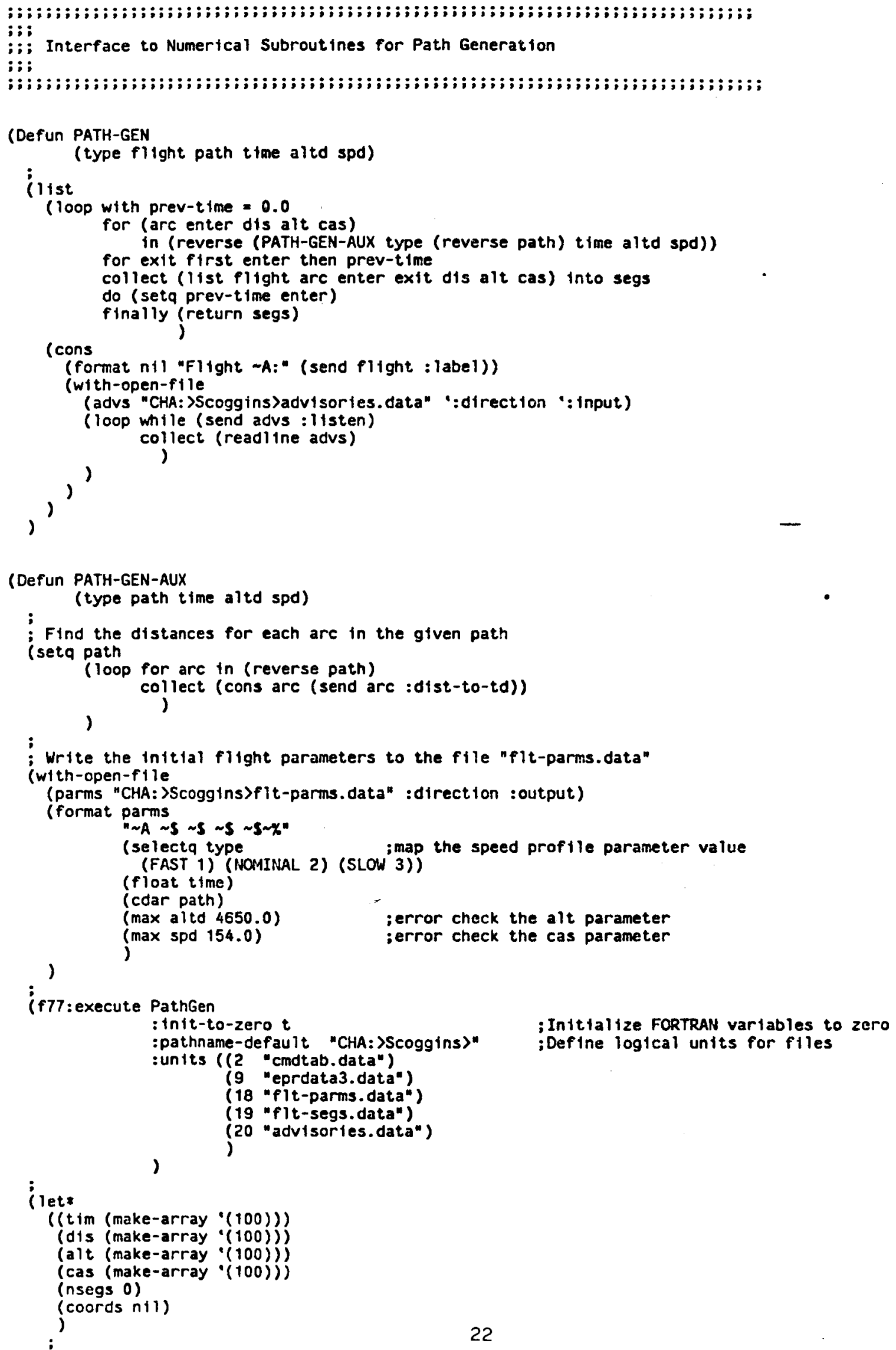

infttalize FORTRAN vartables to zero ;Define logical units for files 


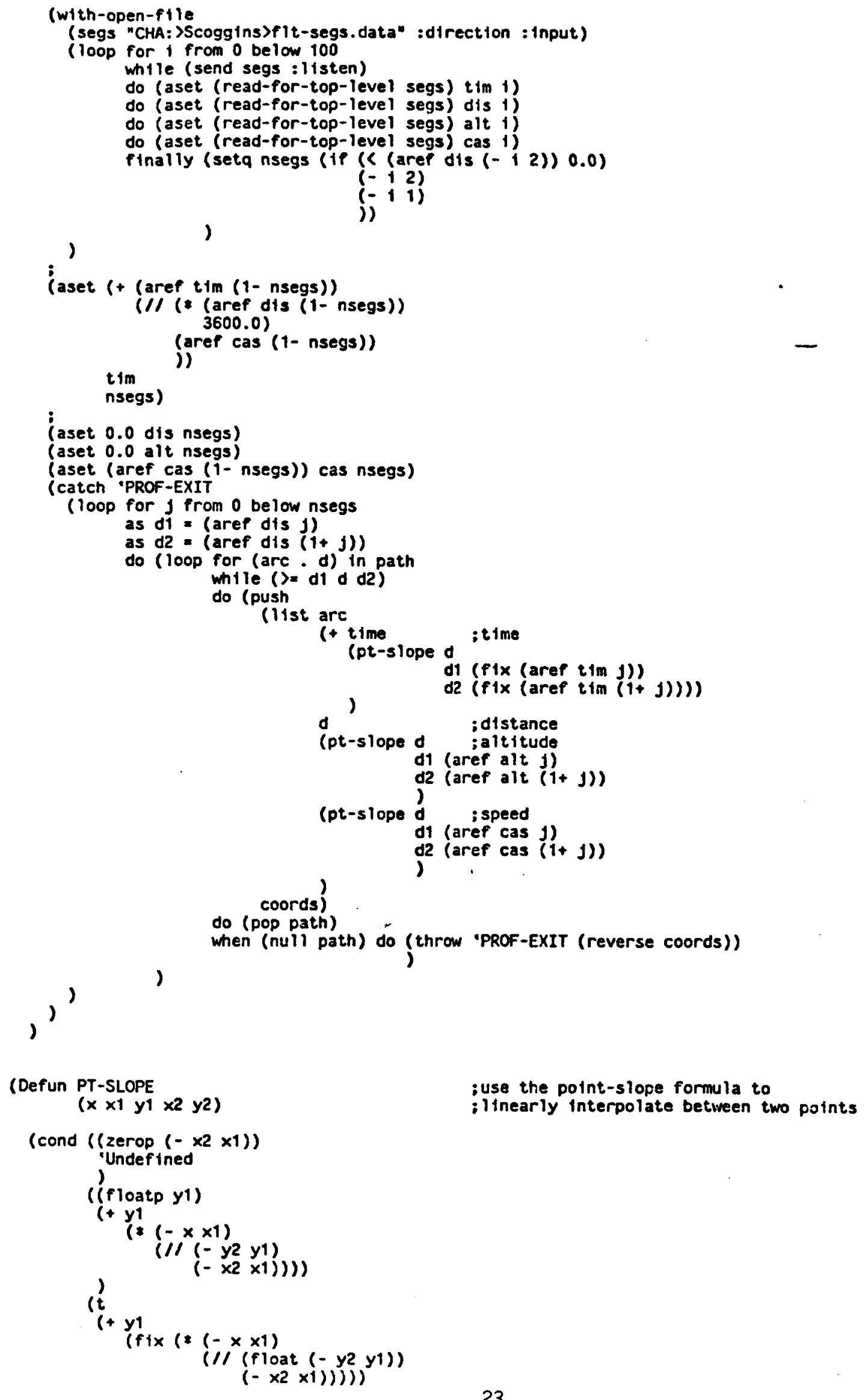


, $)^{\prime}$

$$
\text { ) }
$$




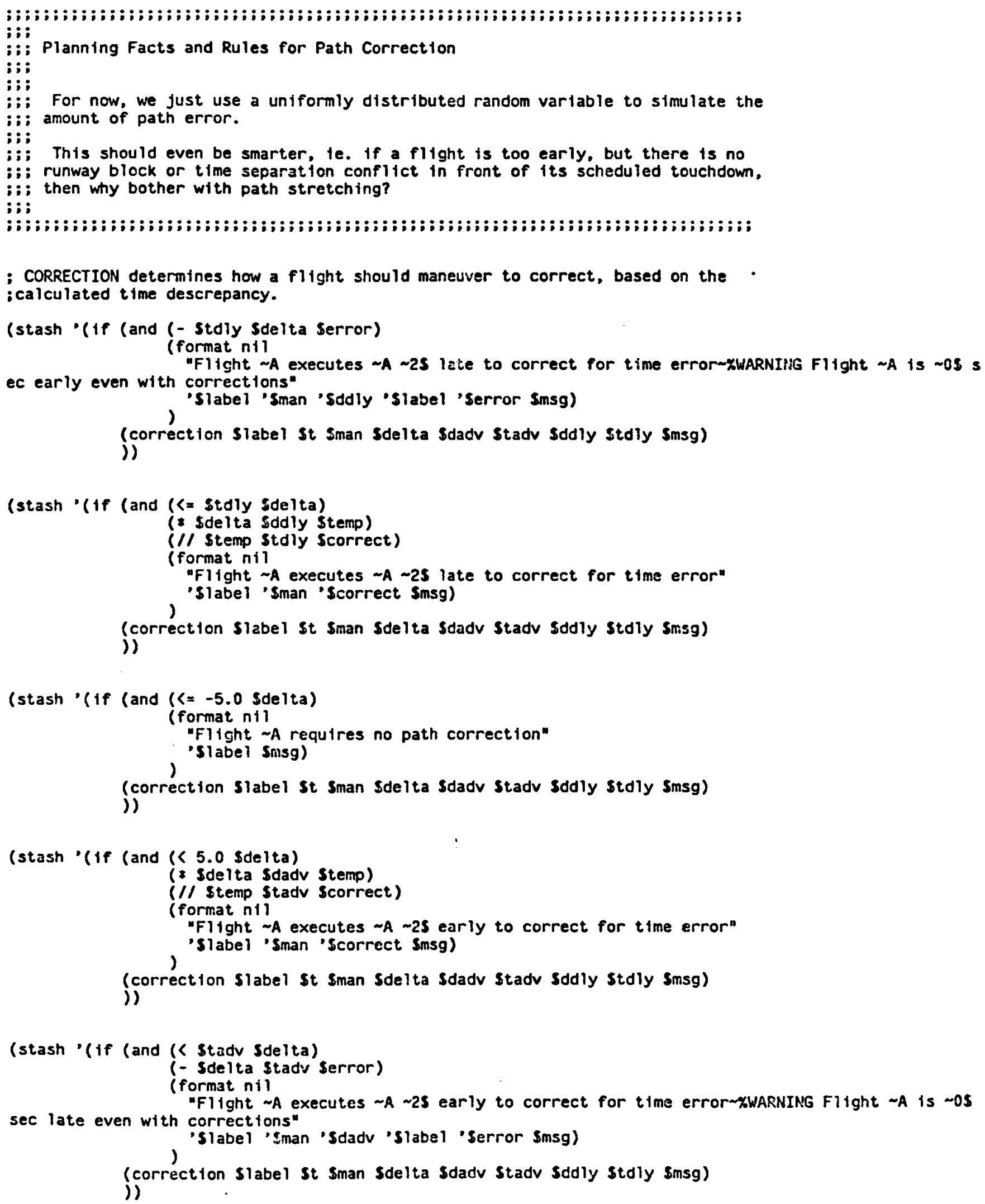




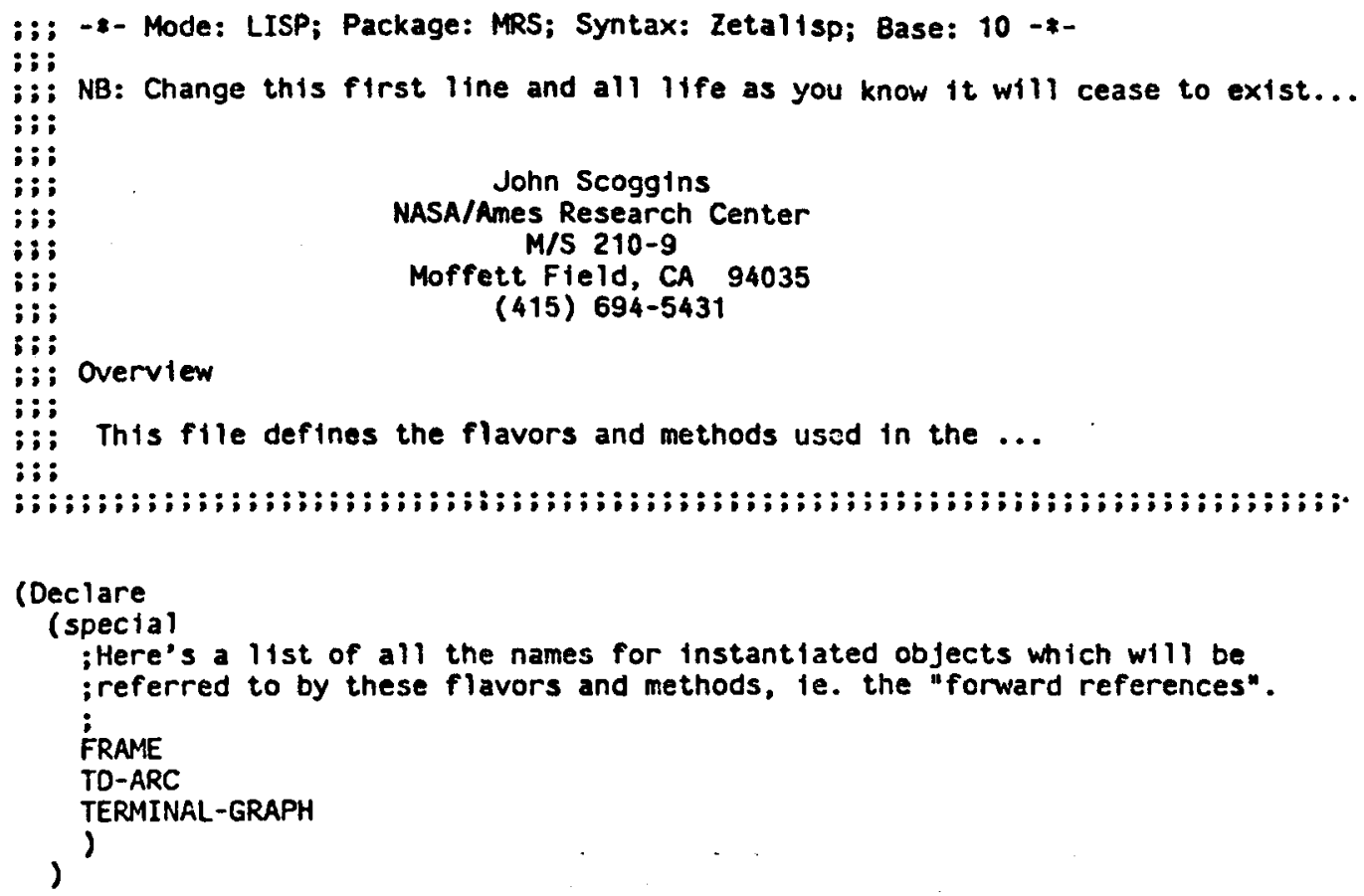




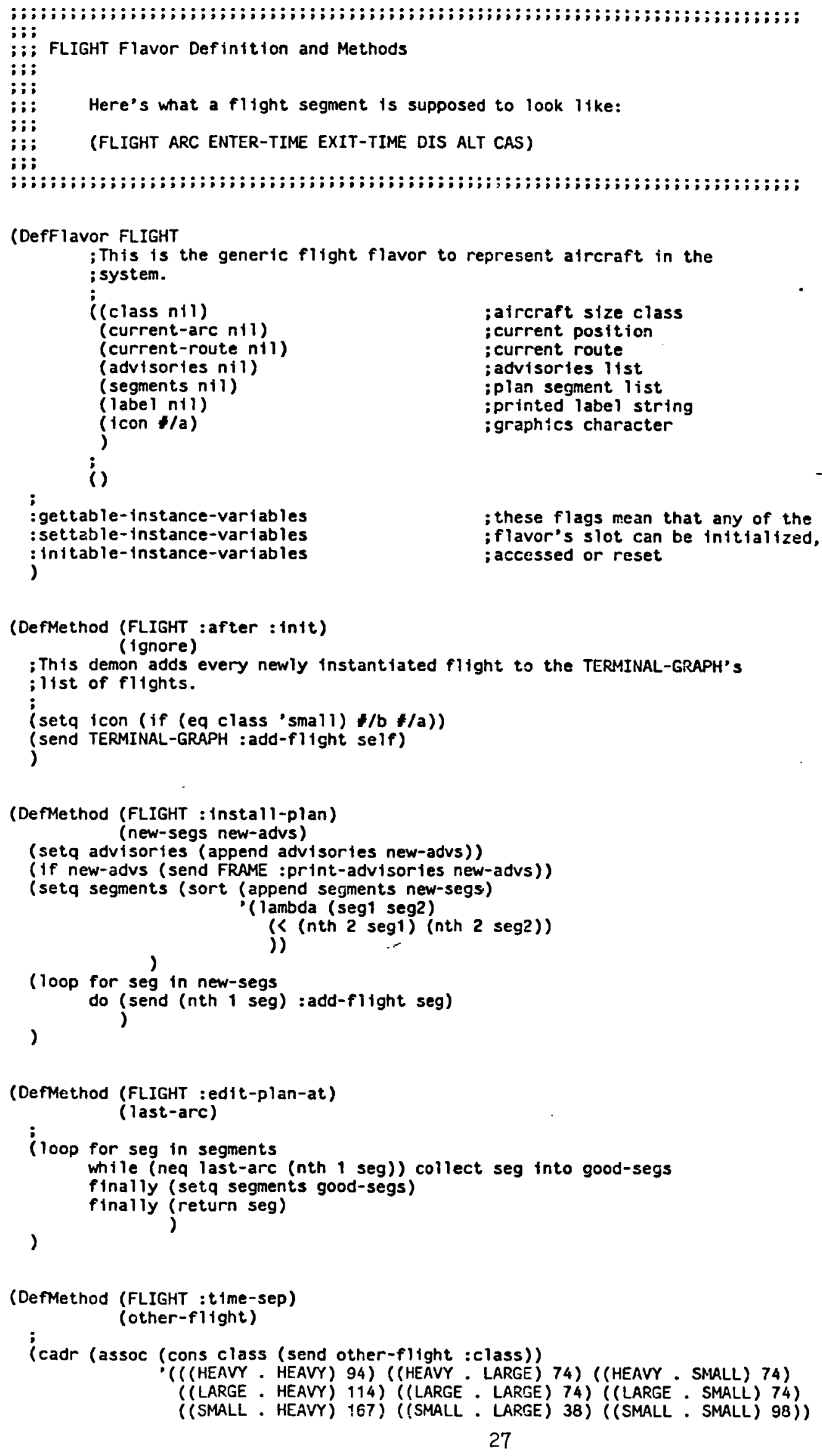




)

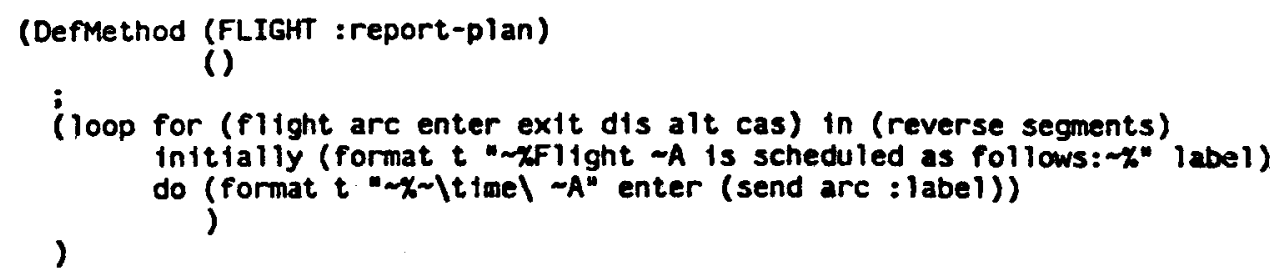




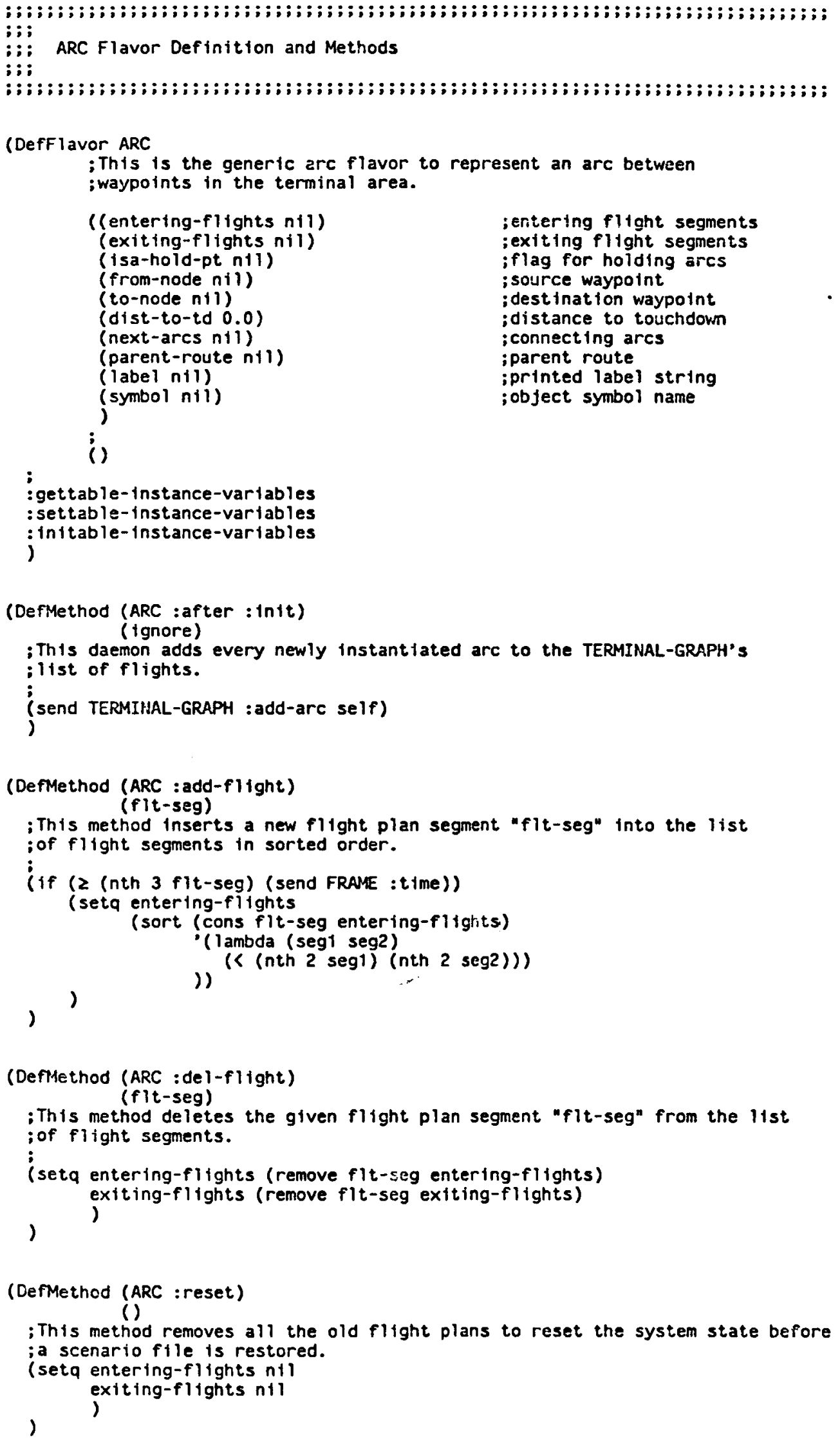




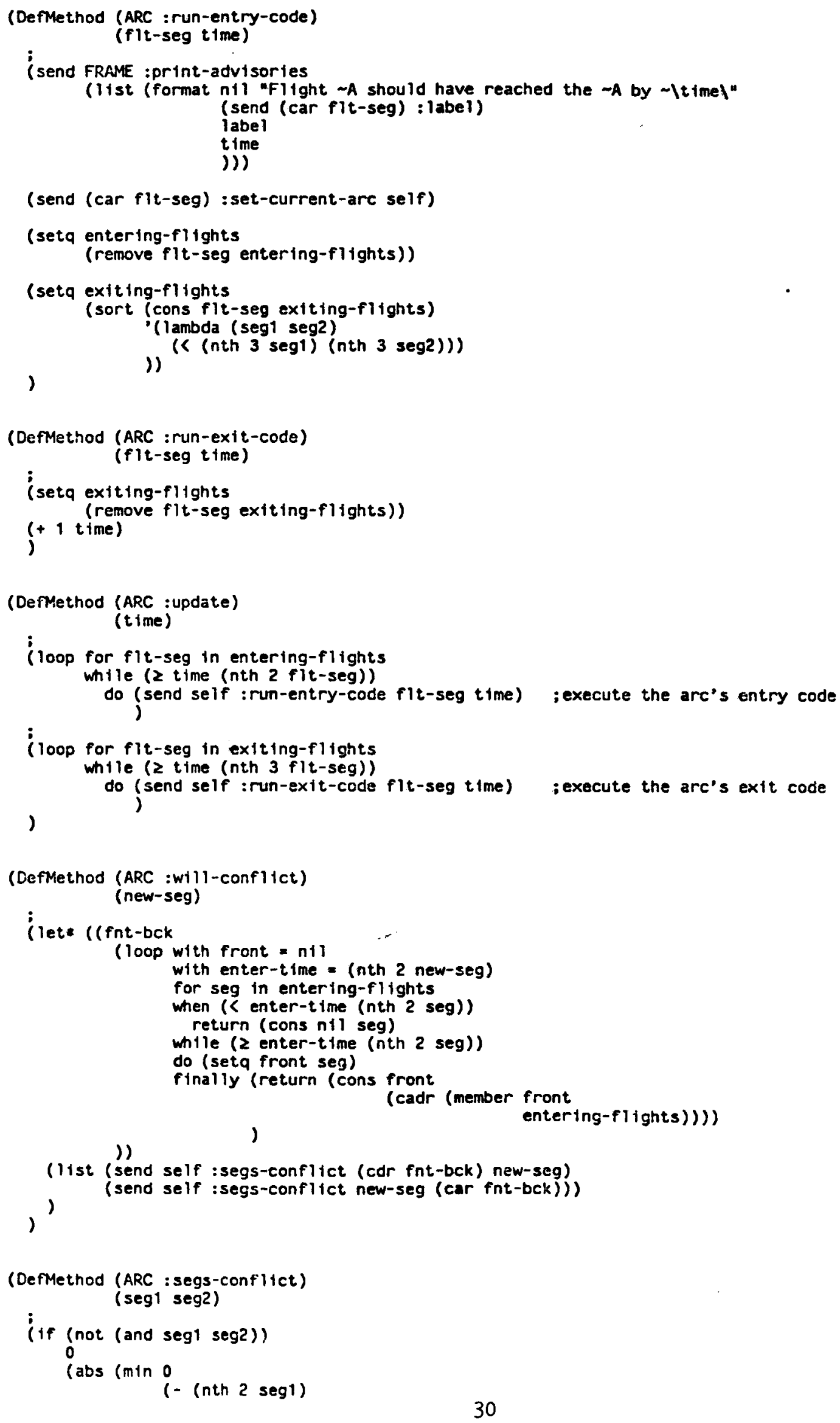


(nth $2 \operatorname{seg} 2)$

(send (car seg1) : tinie-sep (car seg2))

)

)) 


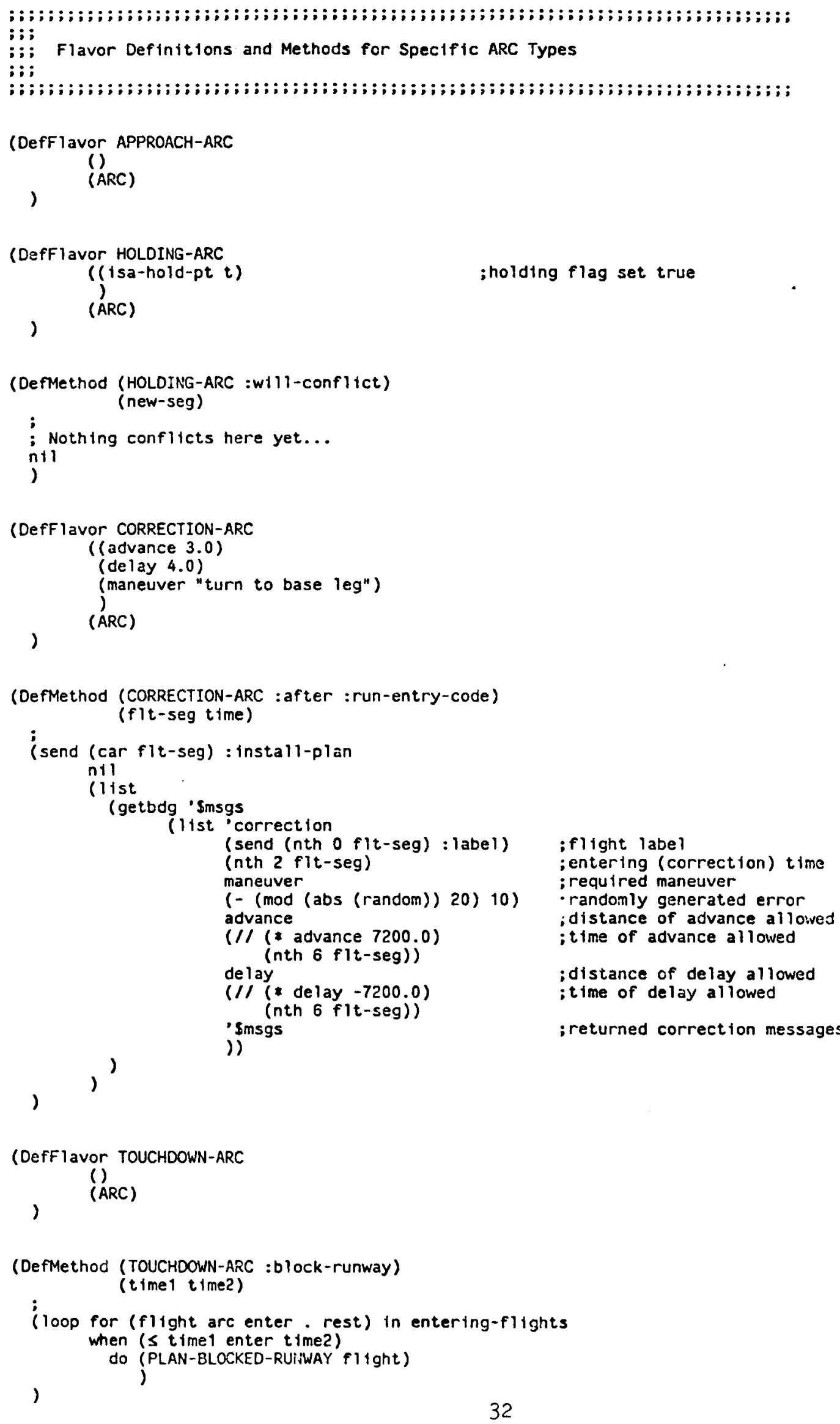




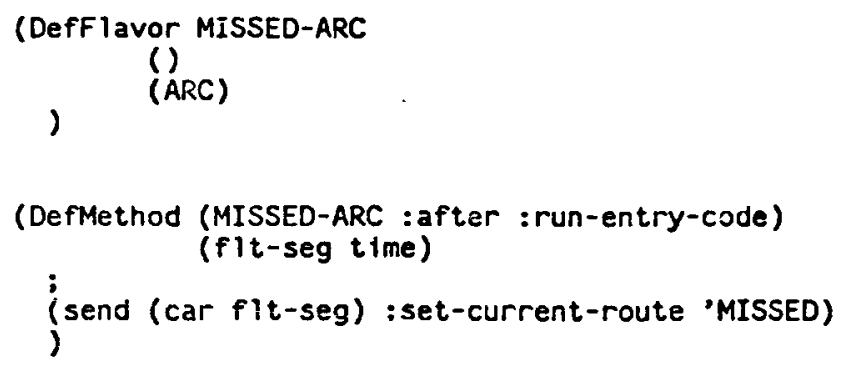




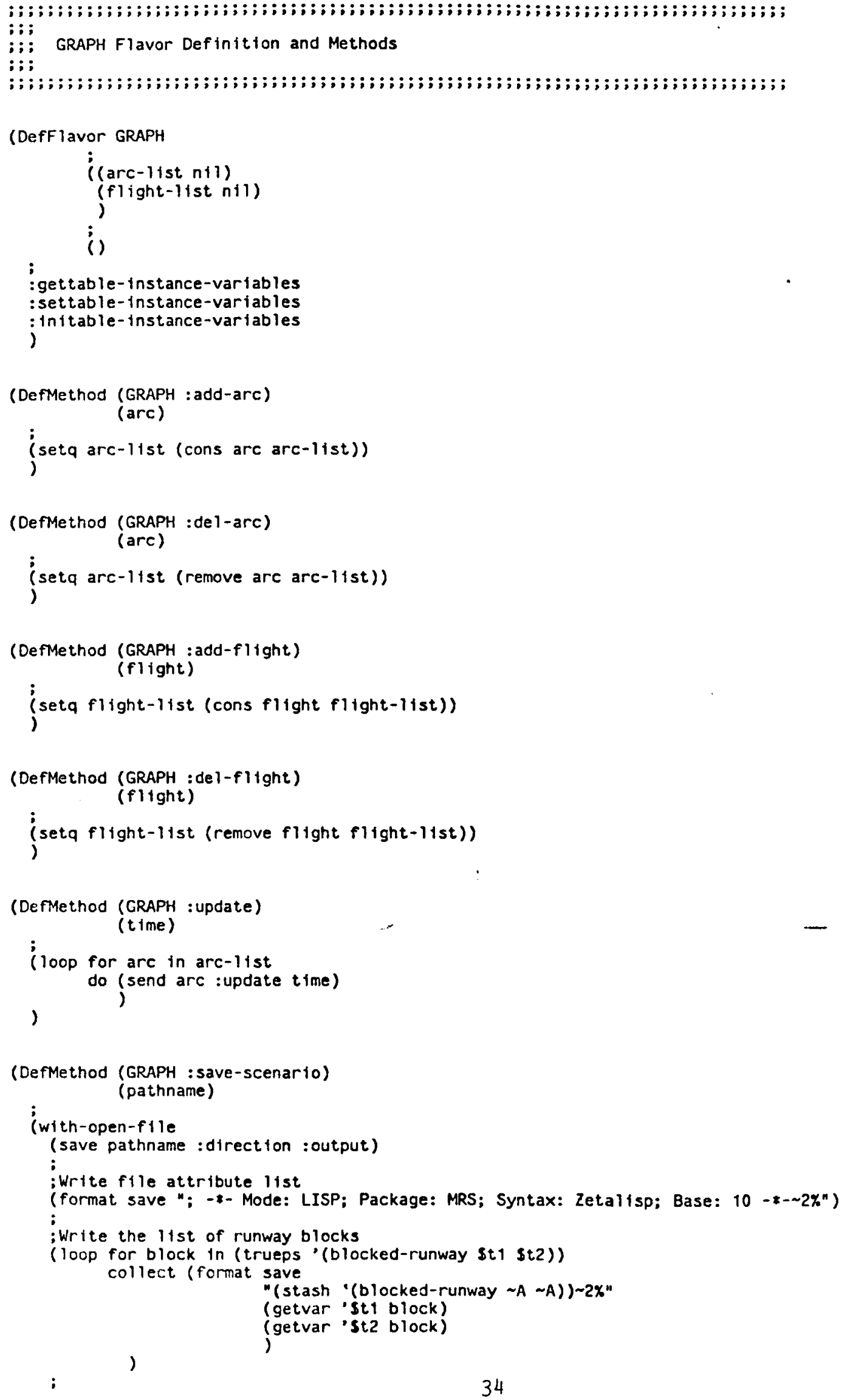




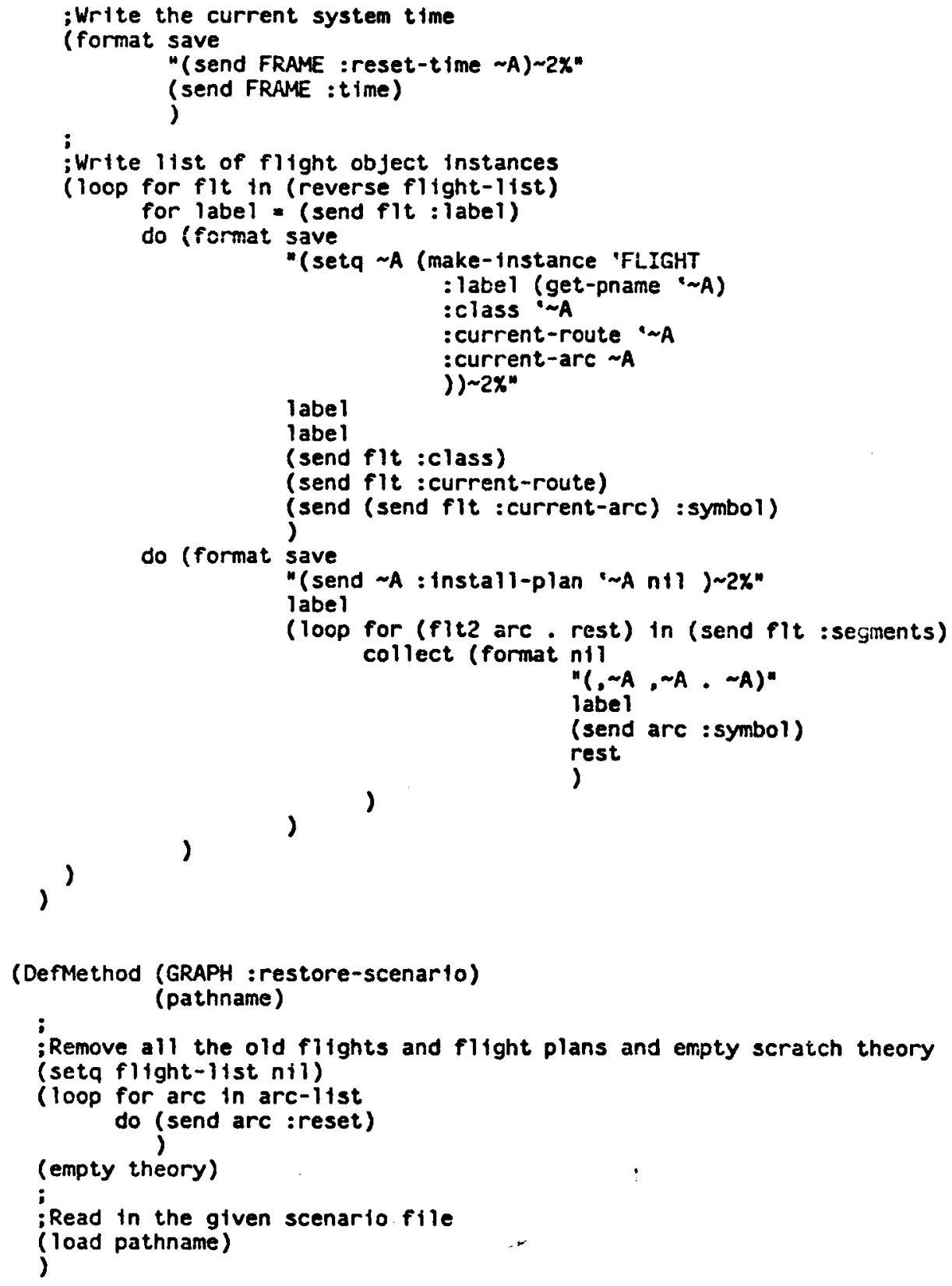




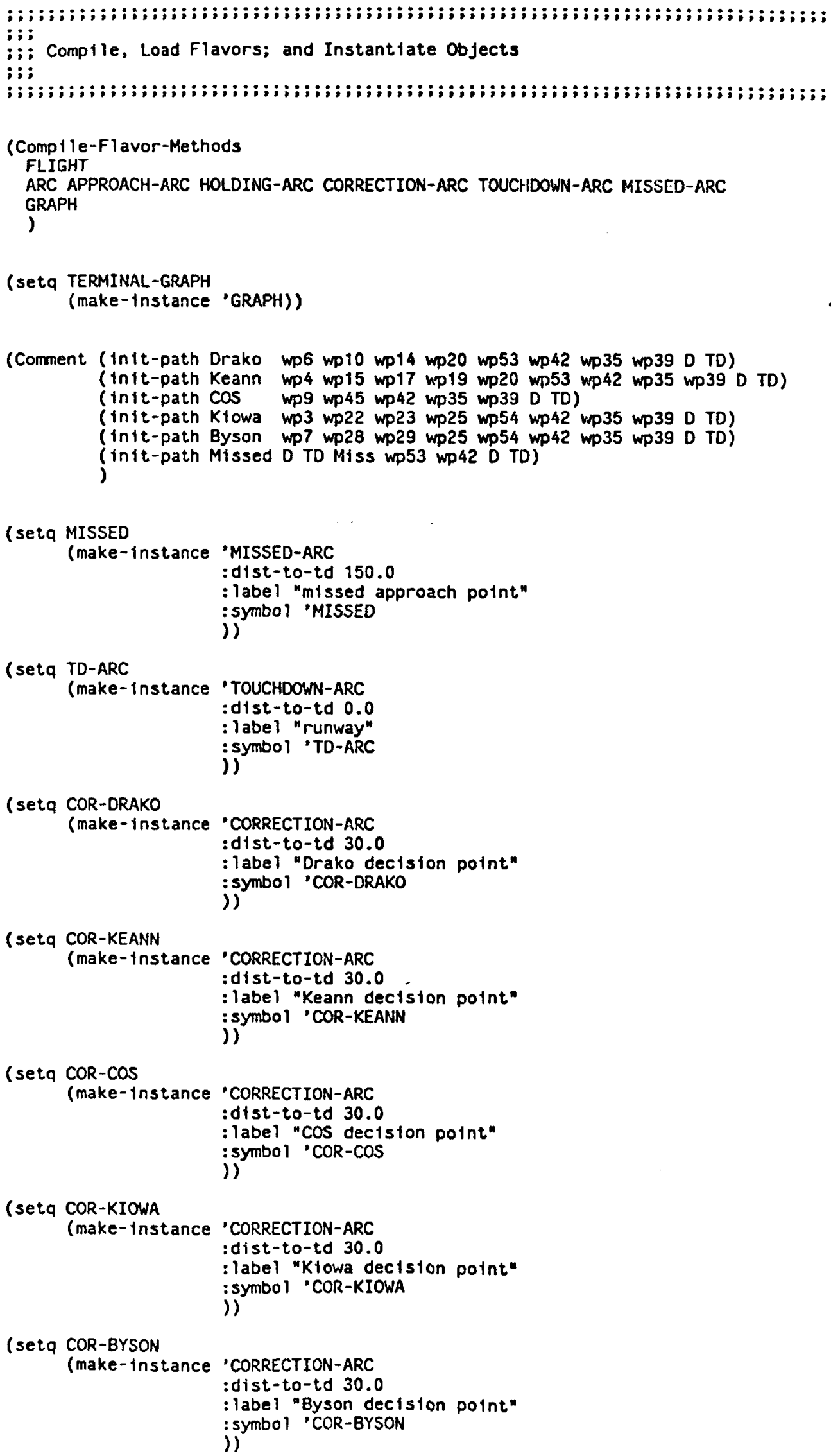

(seta COR-DRAKO

(make-instance 'CORRECTION-ARC :dist-to-td 30.0 
(setq FF-DRAKO

(make-instance 'ARC

:dist-to-td 100.0

: label "Drako feeder $f(x "$

: symbol 'FF-DRAKO

))

(setq FF-KEANN

(make-instance 'ARC

:d1st-to-td 100.0

: label "Keann feeder $f(x$ "

:symbol 'FF-KEANIN

))

(seta FF-cos

(make-instance 'ARC

:dist-to-td 100.0

: label "Cos feeder $f i x "$

: symbol 'FF-COS

I)

(setq FF-KIOWA

(make-1nstance 'ARC

:dist-to-td 100.0

: label "Klowa feeder $f i x$ " : symbol 'FF-KIOWA

i)

(setq FF-BYSON

(make-Instance 'ARC

:dfst-to-td 100.0

:label "Byson feeder fix" :symbol 'FF-BYSON

))

(setq HOLD-DRAKO

(make-instance 'HOLOING-ARC

:dist-to-td 100.0

:label "Orako holding polnt" : symbol 'HOLO-DRAKO

))

(setq HOLD-KEANN

(make-instance 'HOLDING-ARC

:dist-to-td 100.0

:label "Keann holding point"

: symbol 'HOLD-KEANN

))

(setq HOLD-COS

(make-instance 'HOLDING-ARC

:dist-to-td 100.0

:label "Cos holdtng point"

: symbol'HOLD-cos

))

(setq HOLD-KIOWA

(make-instance 'HOLDING-ARC

:dist-to-td 100.0

: label "Klowa holding point"

: symbol 'HOLD-KIOWA

))

(seta HOLD-BYSON

(make-instance 'HOLDING-ARC

:dist-to-td 100.0

:label "Byson holding point"

:symbol 'HOLD-BYSON

i)

(setq APP-DRAKO

(make-instance 'APPROACH-ARC

:dist-to-td 100.0

:label "Drako approach path"

: symbol' APP-DRAKO 
)

(setq APP-KEANN

(make-1nstance 'APPROACH-ARC

:dist-to-td 100.0

: label "Keann approach path"

: symbol 'APP-KEANN

i)

(setq APP-COS

(make-instance 'APPROACH-ARC

:dist-to-td 100.0

:label "COS approach path"

:symbol 'APP-COS

))

(setq APP-KIOWA

(make-Instance 'APPROACH-ARC

:dist-to-td 100.0

:label "Kiowa approach path"

:symbol 'APP-KIOWA

))

(setq APP-BYSON

(make-instance 'APPROACH-ARC

:dist-to-td 100.0

:label "Byson approach path"

:symbol 'APP-BYSON

i) 


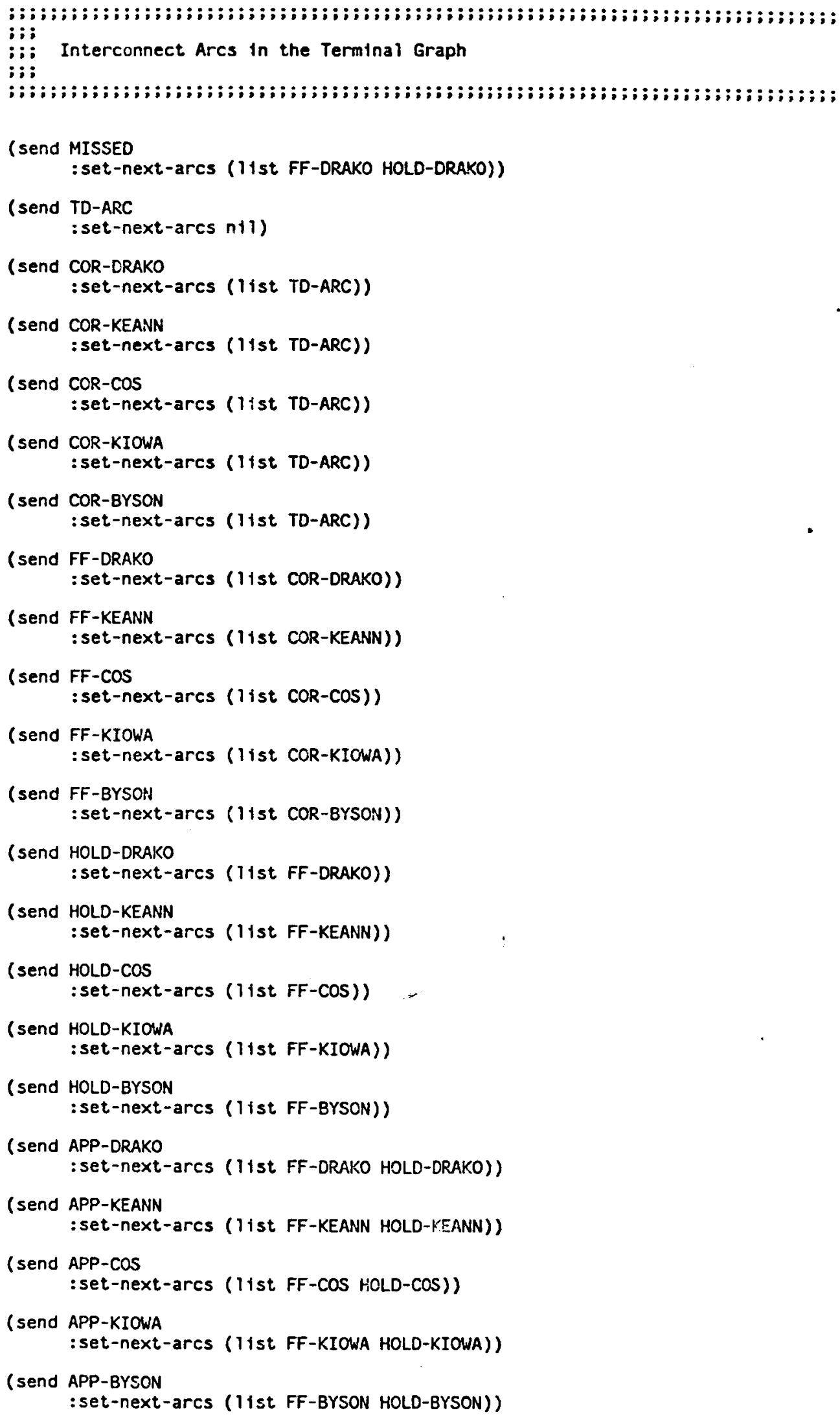




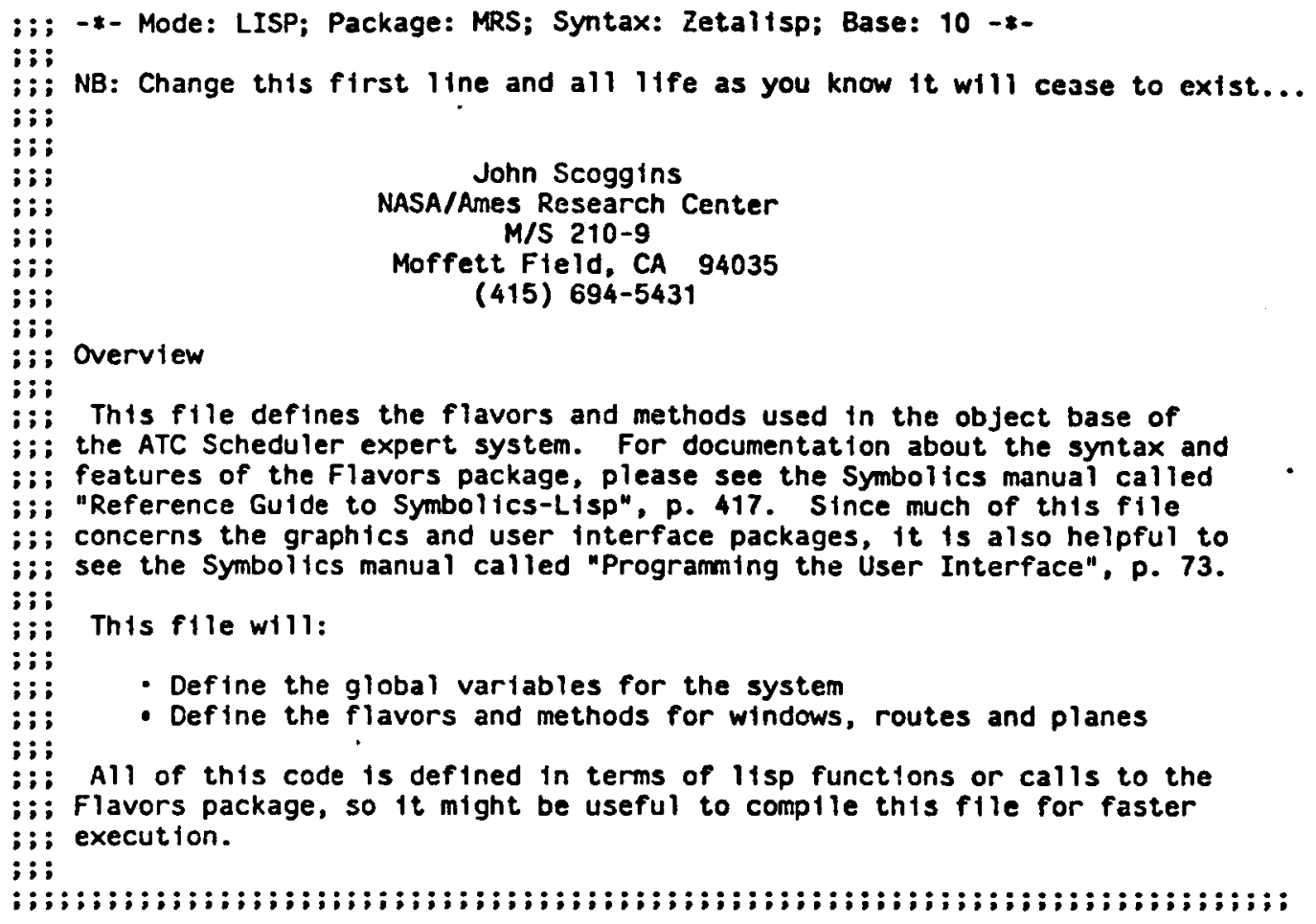




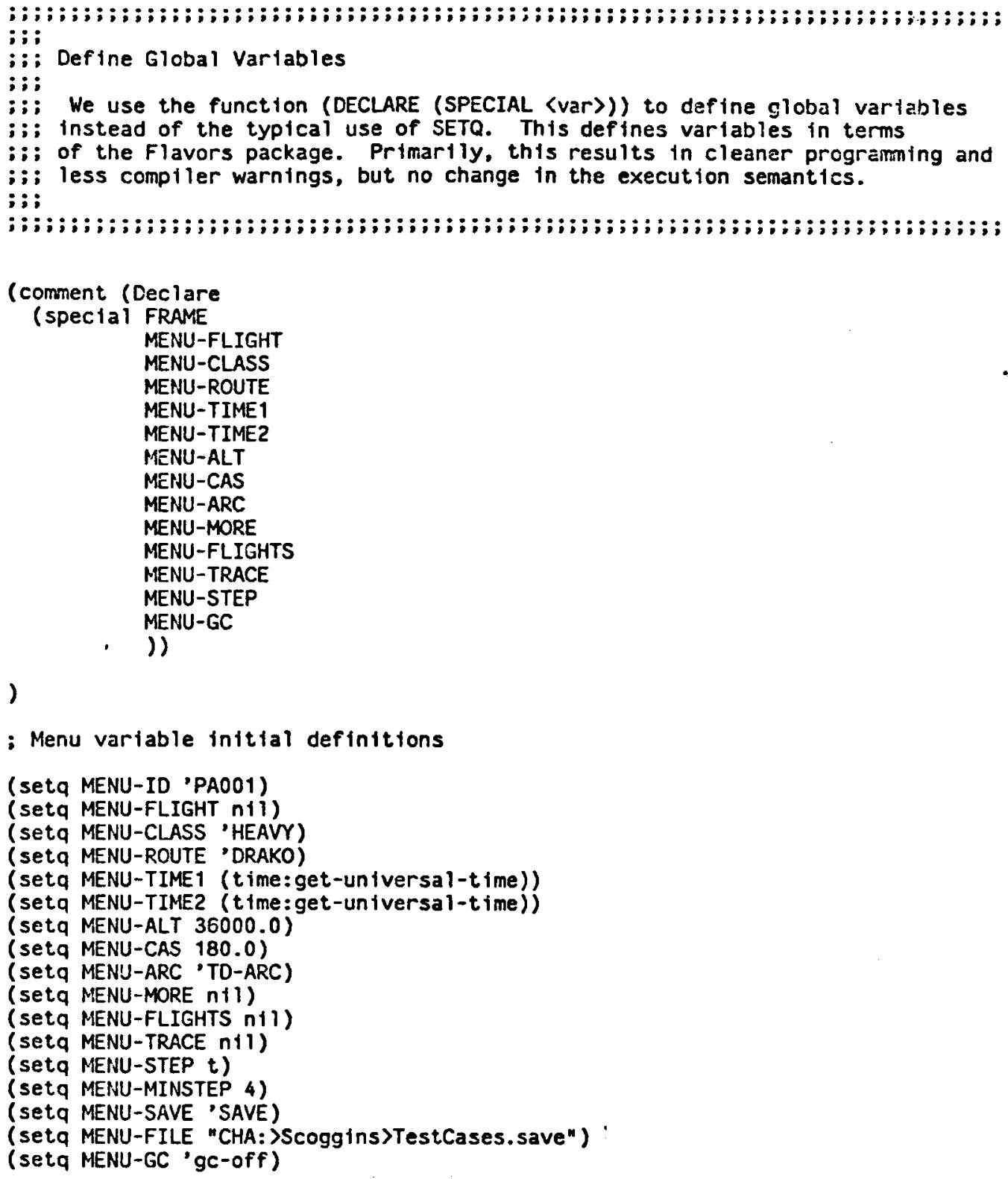




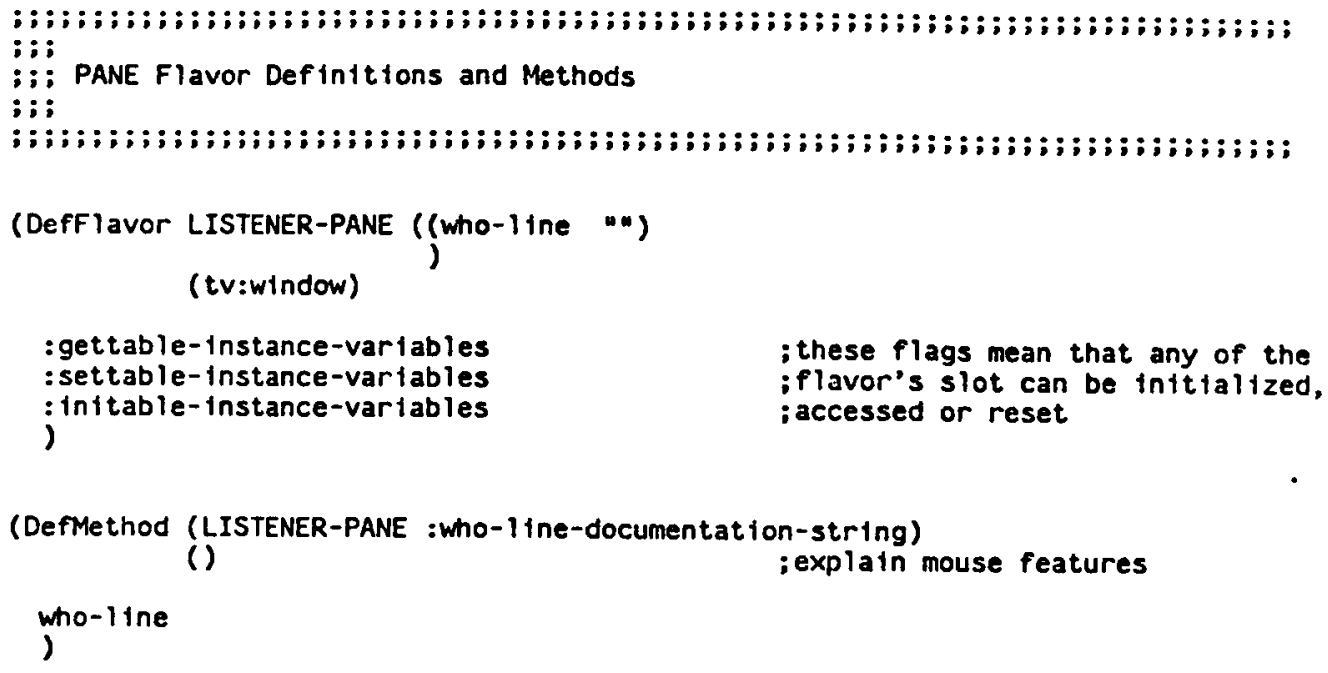




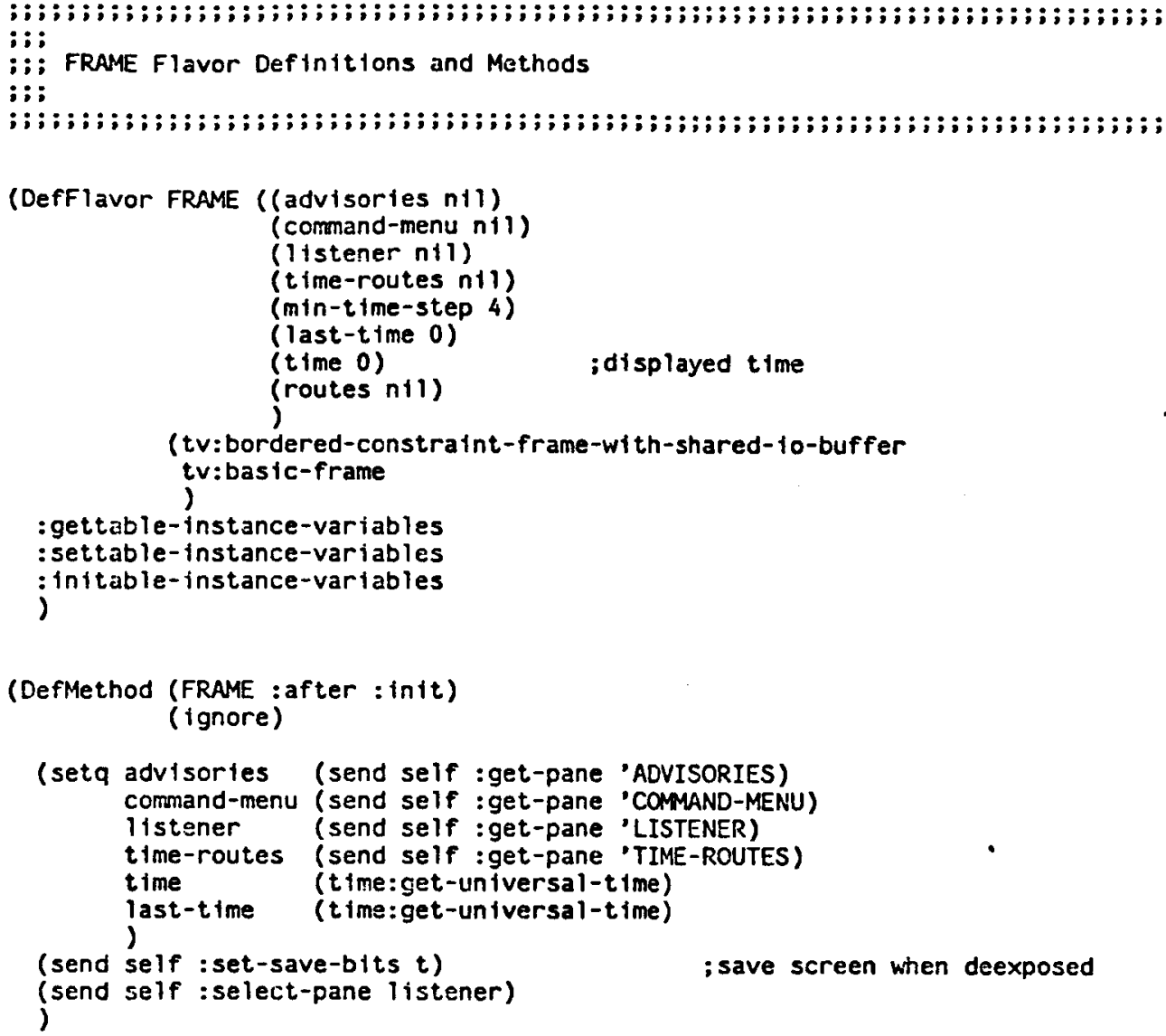

(DefMethod (FRAME : command-loop)

()

; command loop for system - this enables ; the use of mouse/menu drtven user input

(loop do (catch-error-restart ((ERROR SYS:ABORT) "Return to the ATC Scheduler prompt")

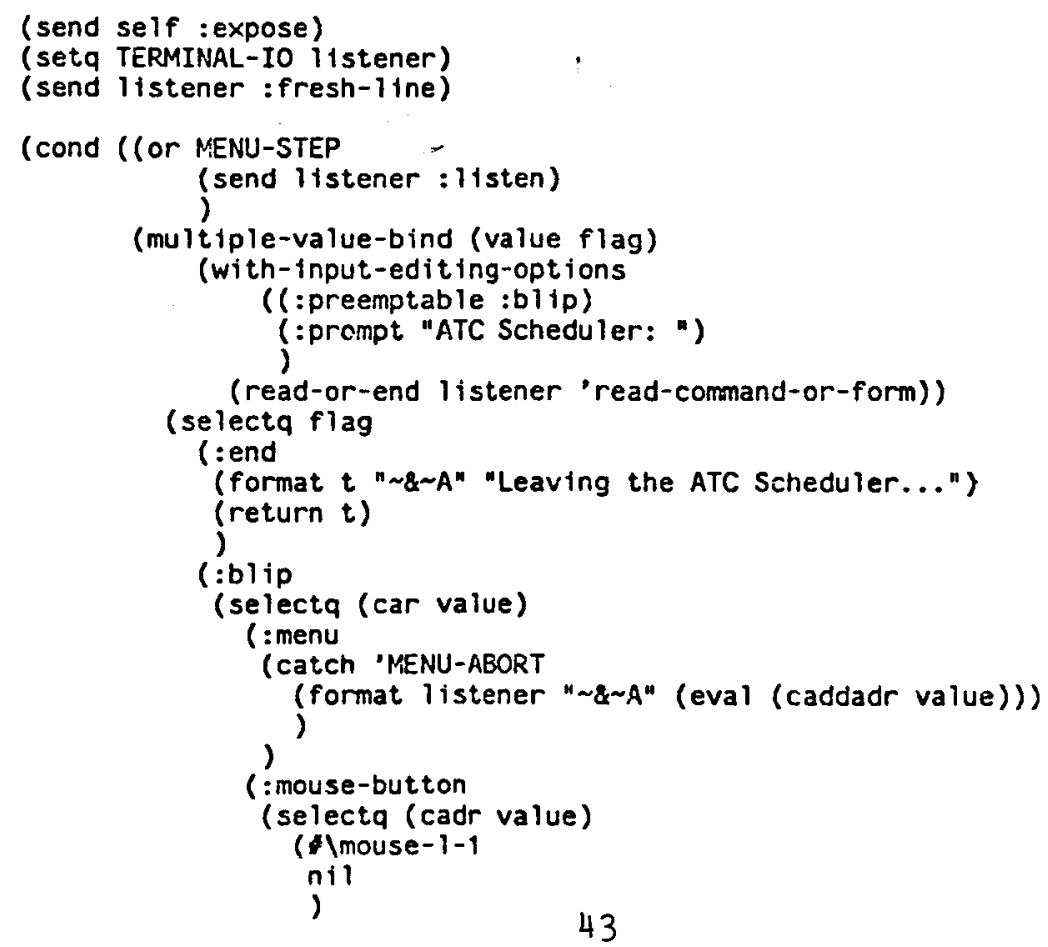




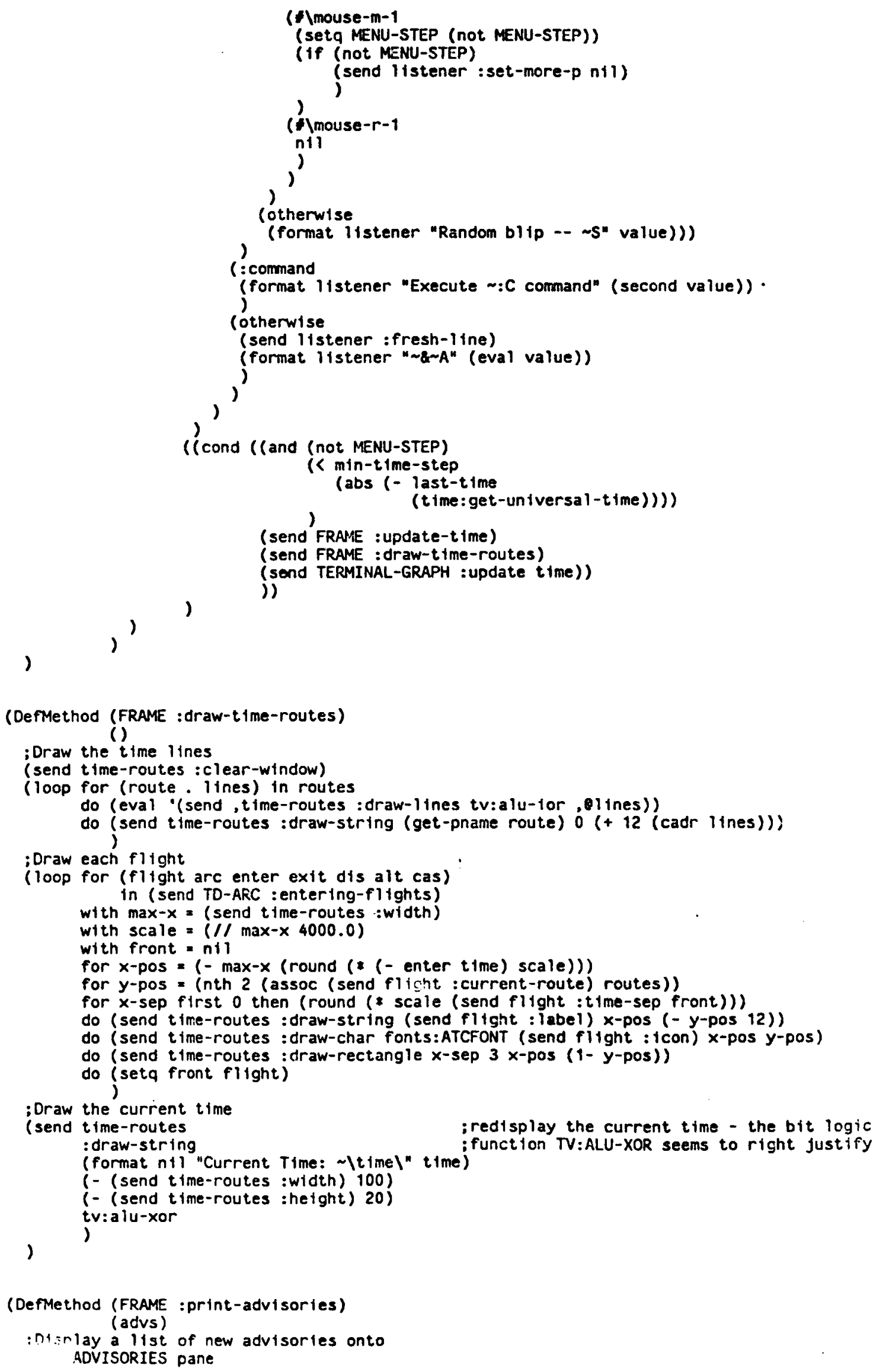




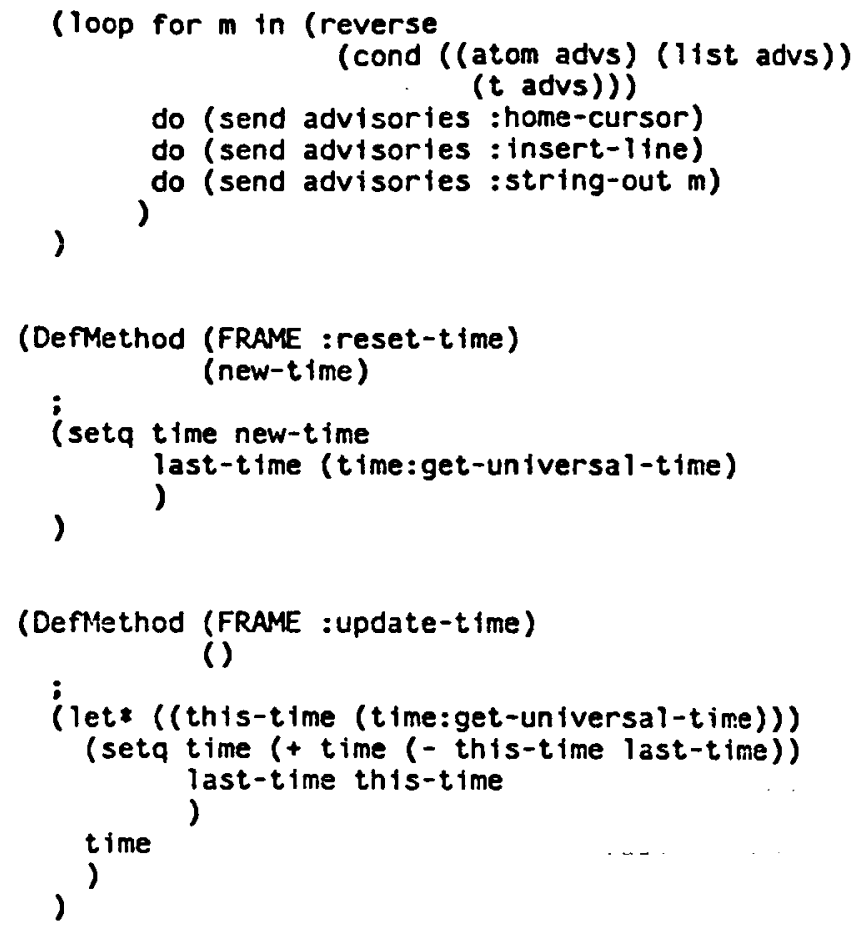




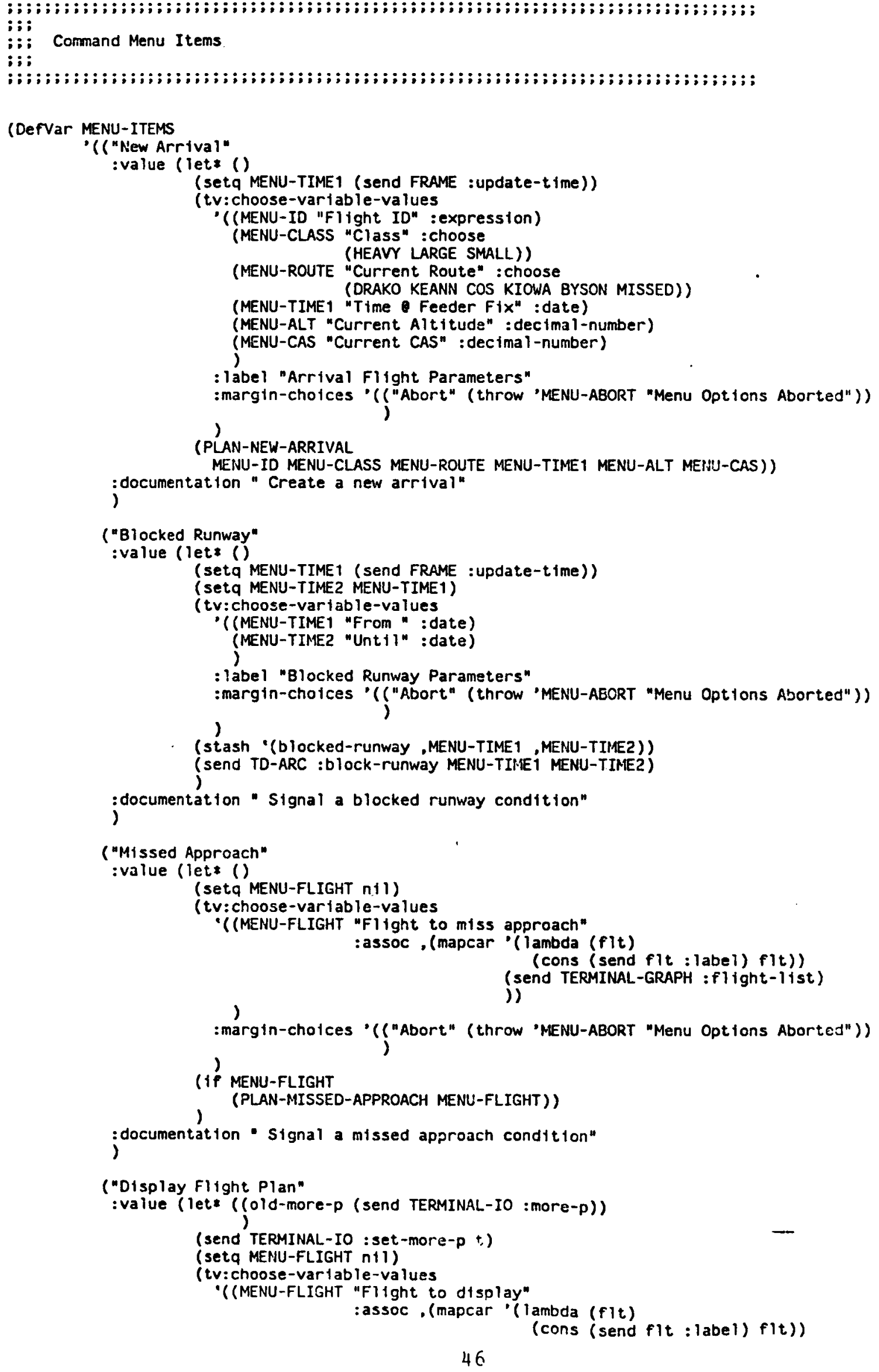




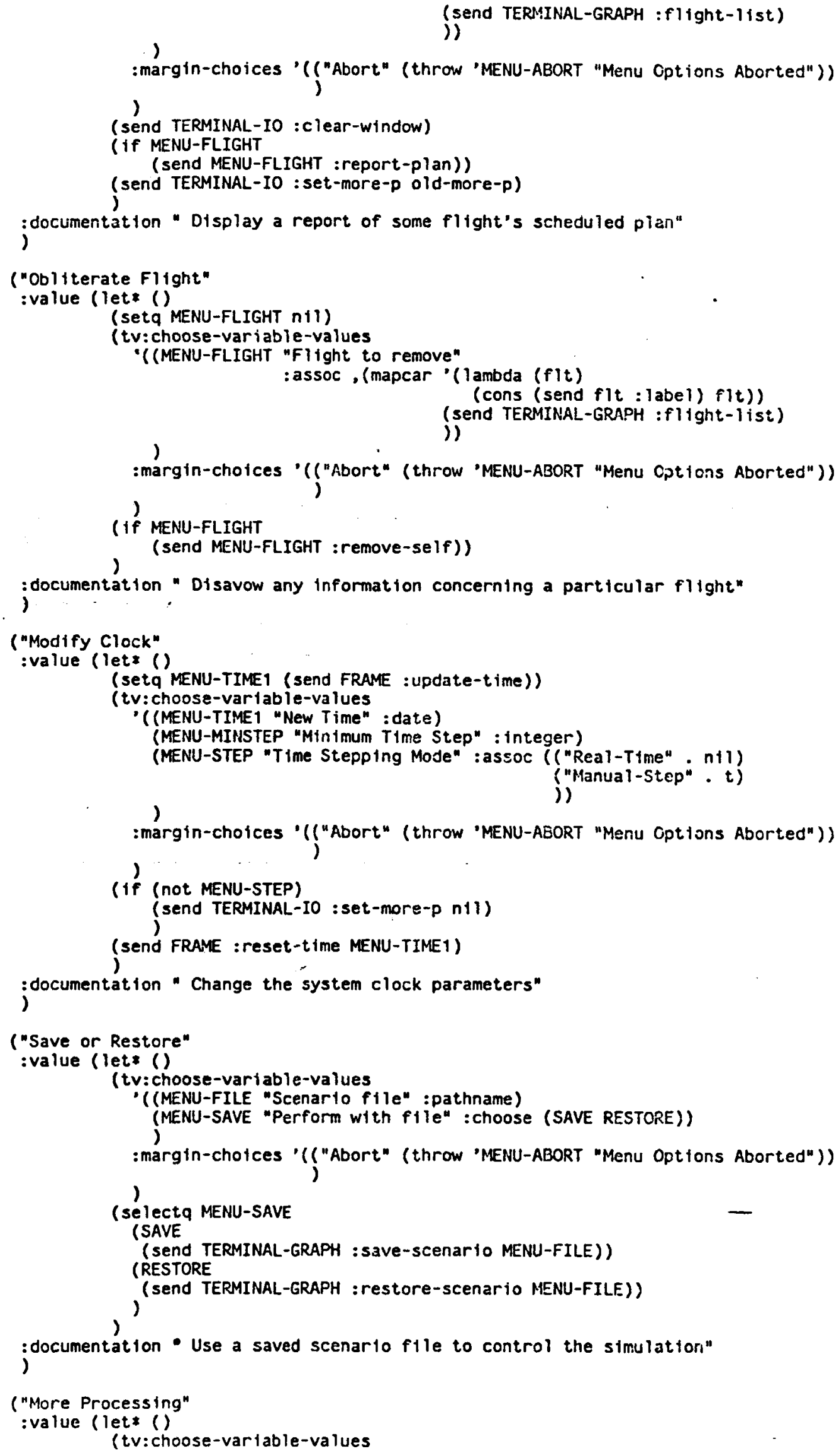




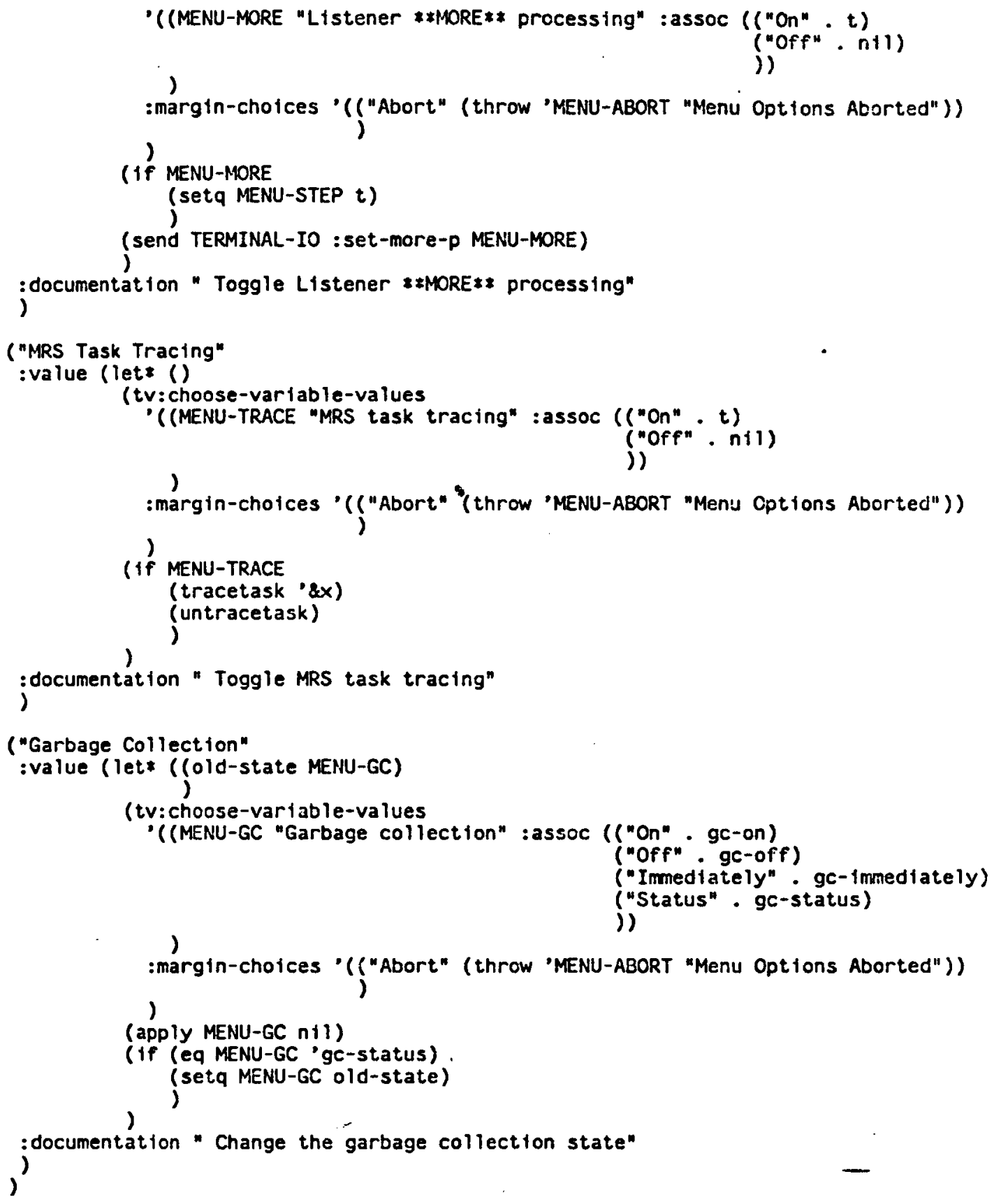




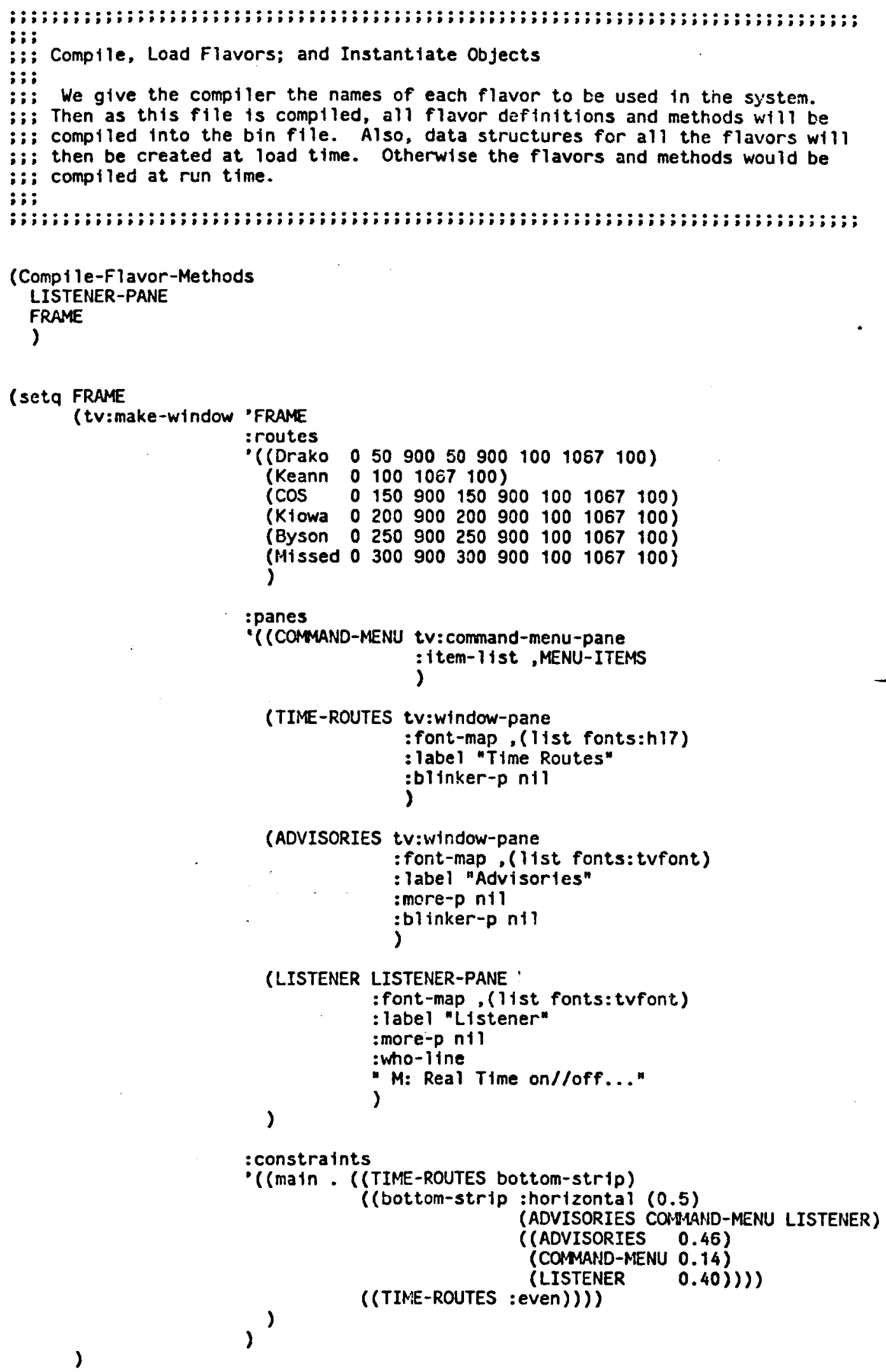




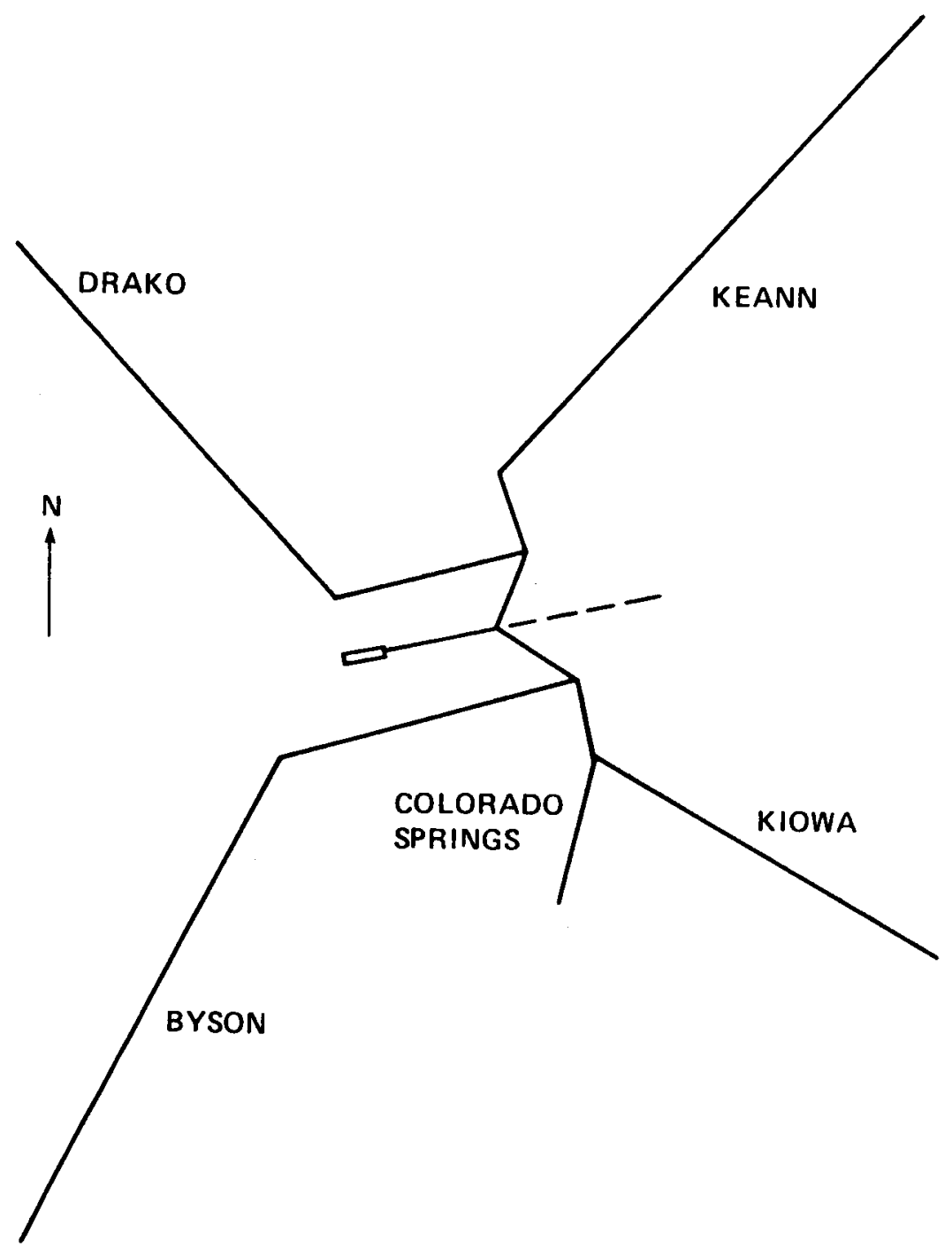

Fig. 1 Denver Route Structure. 


\begin{tabular}{|l|l|}
\hline \multicolumn{3}{|c|}{ WA374 } \\
\hline DRAKO & \\
\hline KEANN & \\
\hline KIOWA & \\
\hline BYSON & \\
\hline MISSED & CURRENT TIME: 12:37:03 \\
\hline
\end{tabular}

Fig. 2 Schedule for New Arrival.

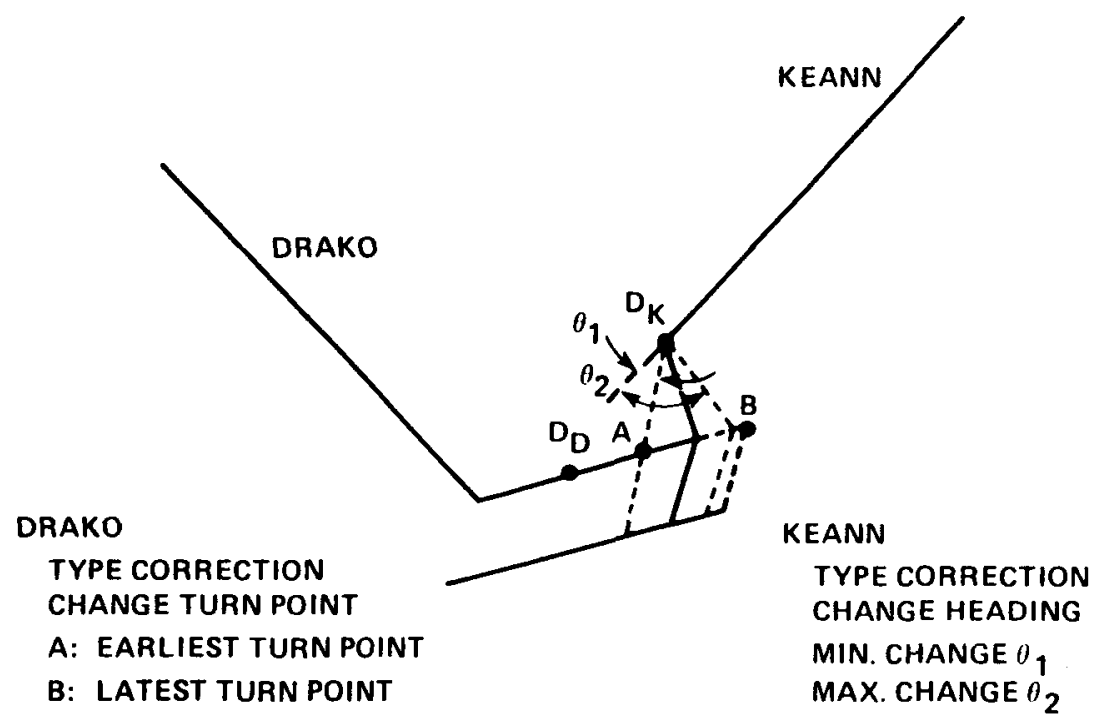

Fig. 3 Path Correction. 


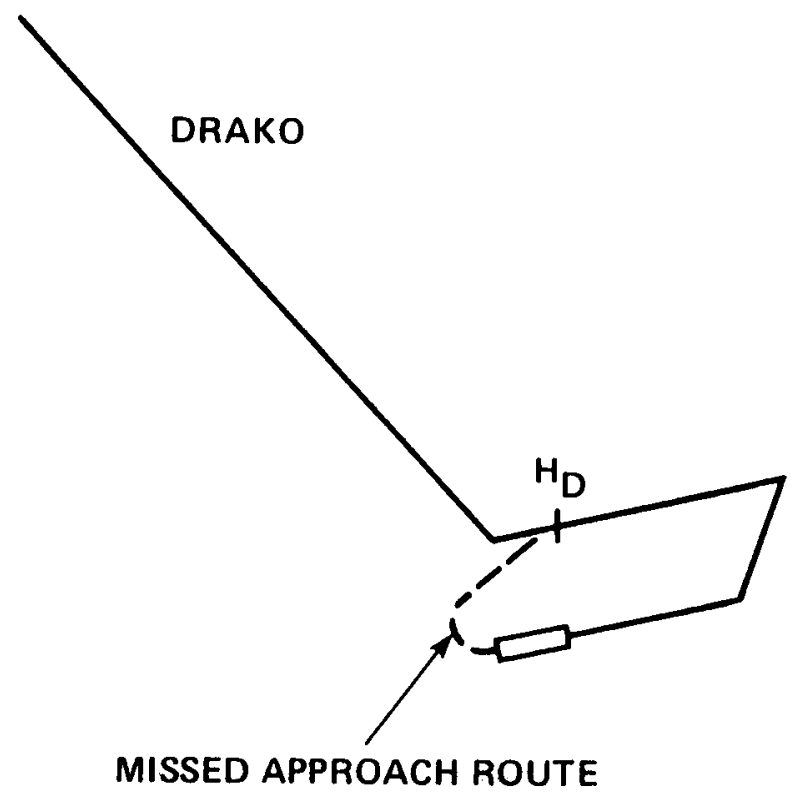

Fig. 4 Missed Approach Route. 


\begin{tabular}{|l|}
\hline DRAKO \\
\hline KEANN \\
\hline KIOWA \\
\hline BYSON \\
\hline MISSED \\
TIME ROUTES \\
\hline
\end{tabular}

(a)

\begin{tabular}{|l|l|}
\hline DRAKO & \\
\hline KEANN & \\
\hline KIOWA & \\
\hline BYSON & \\
\hline MISSED & CURERENT TIME: 13:09:05 \\
\hline
\end{tabular}

(b)

Fig. 5 Time Line. (a) Just before Missed Approach for AA251. (b) Missed Approach for AA251. 


\begin{tabular}{|l|l|}
\hline DRAKO & \\
\hline KEANN & \\
\hline COS & \\
\hline KIOWA & \\
\hline BYSON & \\
\hline MISSED & \\
TIME ROUTES & \\
\hline
\end{tabular}

(a)

\begin{tabular}{|l|c|}
\hline DRAKO & \\
\hline KEANN & \\
\hline KIOW & \\
\hline BYSON & \\
\hline MISSED & \\
TIME ROUTES & CU35 \\
\hline
\end{tabular}

(b)

Fig. 6 Time Line. (a) Runway Closure. (b) Revised Schedule Due to Runway Closure. 


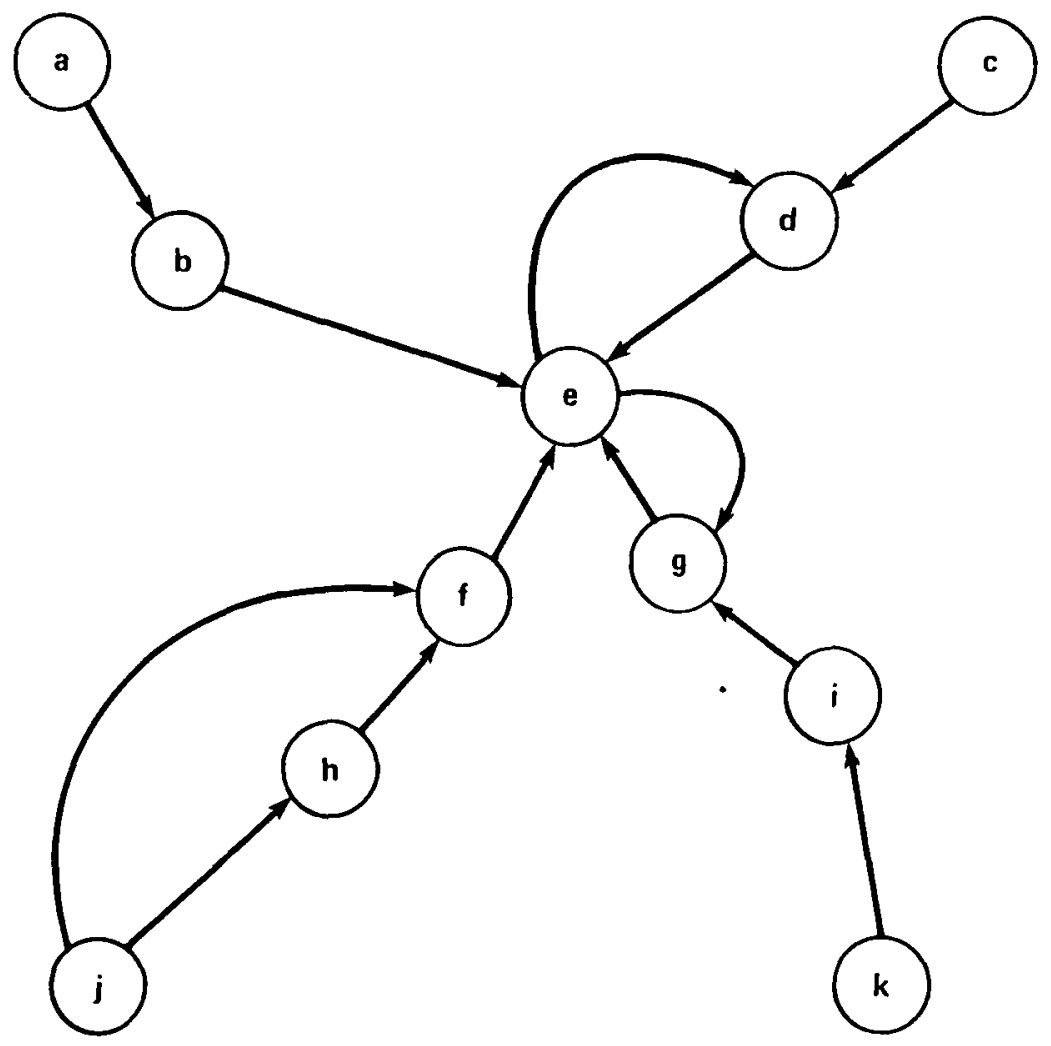

INFORMATION FOR ARC $\mathrm{J} \rightarrow \mathrm{H}$

DISTANCE-TO-TOUCHDOWN: 165 n.mi.

ALTERNATE-PATH: $J \rightarrow F$

ENTRY-CODE: FEEDER FIX ENTRY PROC

EXIT-CODE: FEEDER FIX EXIT PROC

ENTERING.FLIGHTS: (PA35 13:02:23 13:11:45 36000 182)

(VA07 13:10:55 13:20:0134500 178)

EXITING.FLIGHTS: (AA24 12:50:33 12:59:57 32800 191)

(XX01 12:55:41 13:03:28 29000 193)

Fig. 7 Terminal area represented as a direct graph. 


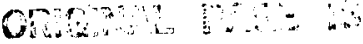 \\ OF POOR QUALITY}

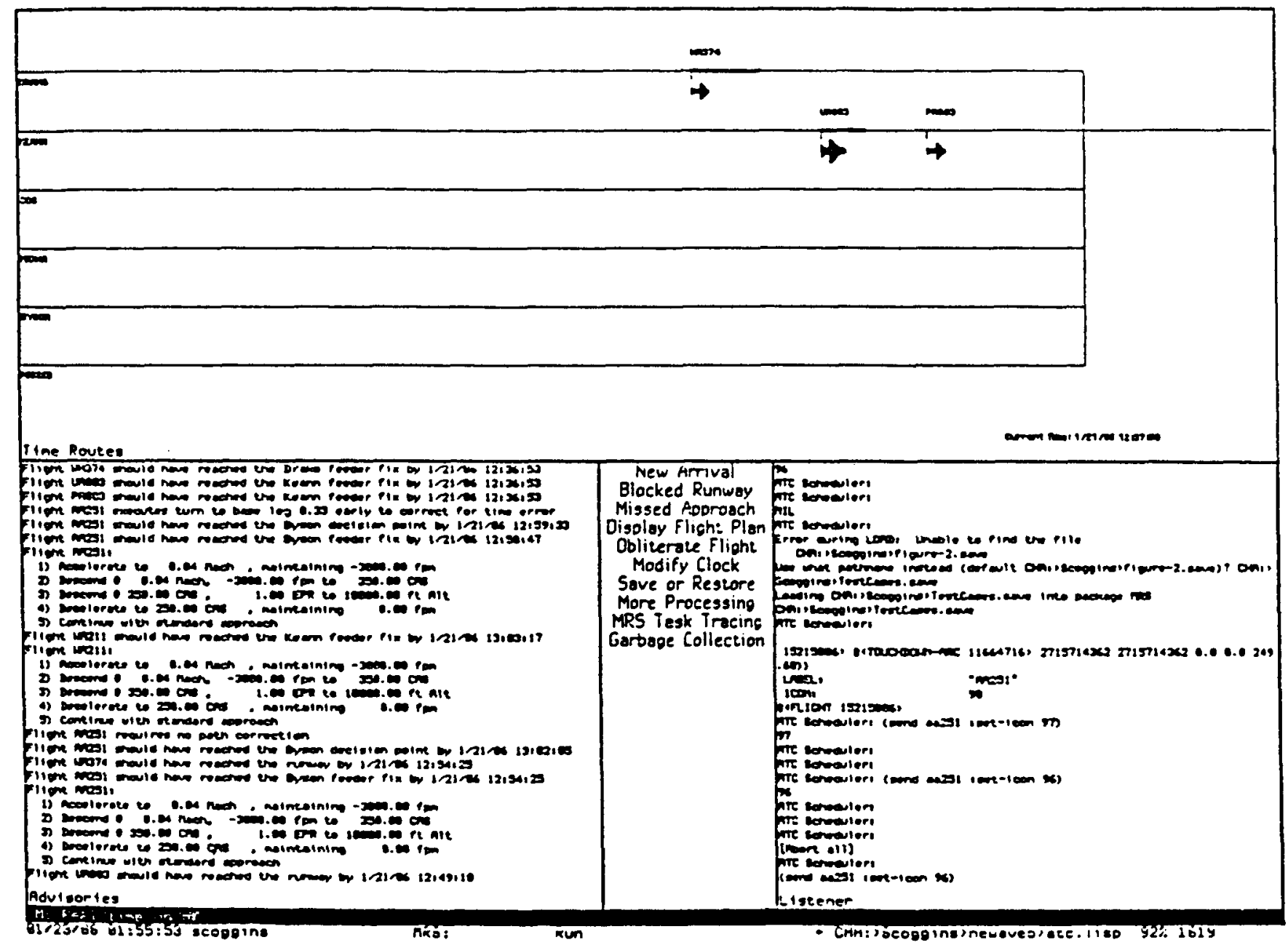

Fig. 8 User interface. 


\begin{tabular}{|c|c|c|c|}
\hline $\begin{array}{l}\text { 1. Aeport No. } \\
\text { NASA TM-88234 }\end{array}$ & 2. Government Accession No. & \multicolumn{2}{|c|}{ 3. Recipient's Catalog No. } \\
\hline \multirow{2}{*}{\multicolumn{2}{|c|}{$\begin{array}{l}\text { 4. Title and Subtitle } \\
\text { TIME-BASED AIR TRAFFIC MANAGEMENT USING } \\
\text { EXPERT SYSTEMS }\end{array}$}} & \multicolumn{2}{|c|}{$\begin{array}{l}\text { 5. Report Date } \\
\text { April } 1986 \\
\end{array}$} \\
\hline & & \multicolumn{2}{|c|}{ 6. Performing Organization Code } \\
\hline \multicolumn{2}{|c|}{$\begin{array}{l}\text { 7. Author(s) } \\
\text { L. Tobias and J. L. Scoggins }\end{array}$} & \multicolumn{2}{|c|}{$\begin{array}{l}\text { 8. Performing Organization Report No. } \\
\text { A-86188 }\end{array}$} \\
\hline \multirow{2}{*}{\multicolumn{2}{|c|}{$\begin{array}{l}\text { 9. Performing Organization Name and Address } \\
\text { Ames Research Center } \\
\text { Moffett Field, CA } 94035\end{array}$}} & \multicolumn{2}{|l|}{ 10. Work Unit No. } \\
\hline & & \multicolumn{2}{|c|}{ 11. Contract or Grant No. } \\
\hline \multirow{2}{*}{\multicolumn{2}{|c|}{$\begin{array}{l}\text { 12. Sponsoring Agency Name and Address } \\
\text { National Aeronautics and Space Administration } \\
\text { Washington, DC } 20546\end{array}$}} & \multicolumn{2}{|c|}{$\begin{array}{l}\text { 13. Type of Report and Period Covered } \\
\text { Technical Memorandum }\end{array}$} \\
\hline & & \multicolumn{2}{|c|}{$\begin{array}{l}\text { 14. Sponsoring Agency Code } \\
505-66-1\end{array}$} \\
\hline \multicolumn{4}{|c|}{$\begin{array}{l}\text { Point of Contact: L. Tobias, Ames Research Center, M/S 210-9, Moffett } \\
\text { Field, CA } 94035 . \quad \text { (415) } 694-5430 \text { or FTS } 464-5430\end{array}$} \\
\hline \multicolumn{4}{|c|}{$\begin{array}{l}\text { 16. Abstract } \\
\text { A prototype expert system has been developed for the time scheduling } \\
\text { of aircraft into the terminal area. The three functions of the air- } \\
\text { traffic-control schedule advisor are as follows : First, for each new } \\
\text { arrival, it develops an admissible flight plan for that aircraft. Second, } \\
\text { as the aircraft progresses through the terminal area, it monitors devia- } \\
\text { tions from the aircraft's flight plan and provides advisories to return } \\
\text { the aircraft to its assigned schedule. Third, if major disruptions such } \\
\text { as missed approaches occur, it develops a revised plan. The advisor is } \\
\text { operational on a Symbolics } 3600 \text {, and is programmed in MRs (a logic pro- } \\
\text { gramming language), Lisp, and Fortran. }\end{array}$} \\
\hline $\begin{array}{l}\text { 17. Key Words (Suggested by Author(s)) } \\
\text { Air traffic control } \\
\text { Expert system } \\
\text { Scheduling }\end{array}$ & $\begin{array}{ll}\text { 18. } & \text { Distributic } \\
\text { Unlim }\end{array}$ & it & ory -03 \\
\hline $\begin{array}{l}\text { 19. Security Clossif. lof this report) } \\
\text { Unclassified }\end{array}$ & $\begin{array}{l}\text { 20. Security Classif. (of this page) } \\
\text { Unclassified }\end{array}$ & $\begin{array}{l}\text { 21. No. of Pages } \\
58\end{array}$ & $\begin{array}{l}\text { 22. Price" } \\
\text { A04 }\end{array}$ \\
\hline
\end{tabular}

